DESY 04-023

ISSN 0418-9833

NSF-KITP-04-24

hep-ph/0404039

April 2004

\title{
Associated Production of Bottomonia and Higgs Bosons at Hadron Colliders
}

\author{
BERND A. KNIEHL* \\ Kavli Institute for Theoretical Physics, University of California, \\ Santa Barbara, CA 93106-4030, USA \\ Caesar P. Palisoc \\ National Institute of Physics, University of the Philippines, \\ Diliman, Quezon City 1101, Philippines \\ LENNART ZWIRNER \\ II. Institut für Theoretische Physik, Universität Hamburg, \\ Luruper Chaussee 149, 22761 Hamburg, Germany
}

\begin{abstract}
We study the associated production of bottomonia and Higgs bosons at hadron colliders within the factorization formalism of nonrelativistic quantum chromodynamics providing all contributing partonic cross sections in analytic form. While such processes tend to be suppressed in the standard model, they may have interesting cross sections in its minimal supersymmetric extension, especially at large values of $\tan \beta$, where the bottom Yukawa couplings are enhanced. We present numerical results for the processes involving the lighter $C P$-even $h^{0}$ boson and the $C P$-odd $A^{0}$ boson appropriate for the Fermilab Tevatron and the CERN LHC.
\end{abstract}

PACS numbers: 12.60.Jv, 13.85.Fb, 14.40.Gx, 14.80.Cp

*Permanent address: II. Institut für Theoretische Physik, Universität Hamburg, Luruper Chaussee 149, 22761 Hamburg, Germany. 


\section{Introduction}

Since the discovery of the $J / \psi$ meson in 1974 , heavy quarkonium has provided a useful laboratory for quantitative tests of quantum chromodynamics (QCD) and, in particular, of the interplay of perturbative and nonperturbative phenomena. The factorization formalism of nonrelativistic QCD (NRQCD) [1] provides a rigorous theoretical framework for the description of heavy-quarkonium production and decay. This formalism implies a separation of short-distance coefficients, which can be calculated perturbatively as expansions in the strong-coupling constant $\alpha_{s}$, from long-distance matrix elements (MEs), which must be extracted from experiment. The relative importance of the latter can be estimated by means of velocity scaling rules; i.e., the MEs are predicted to scale with a definite power of the heavy-quark $(Q)$ velocity $v$ in the limit $v \ll 1$. In this way, the theoretical predictions are organized as double expansions in $\alpha_{s}$ and $v$. A crucial feature of this formalism is that it takes into account the complete structure of the $Q \bar{Q}$ Fock space, which is spanned by the states $n={ }^{2 S+1} L_{J}^{(a)}$ with definite spin $S$, orbital angular momentum $L$, total angular momentum $J$, and color multiplicity $a=1,8$. In particular, this formalism predicts the existence of color-octet $(\mathrm{CO})$ processes in nature. This means that $Q \bar{Q}$ pairs are produced at short distances in CO states and subsequently evolve into physical, color-singlet (CS) quarkonia by the nonperturbative emission of soft gluons. In the limit $v \rightarrow 0$, the traditional CS model (CSM) [2] is recovered. The greatest triumph of this formalism was that it was able to correctly describe [3-5] the cross section of inclusive charmonium hadroproduction measured in $p \bar{p}$ collisions at the Fermilab Tevatron [6], which had turned out to be more than one order of magnitude in excess of the theoretical prediction based on the CSM.

Apart from this phenomenological drawback, the CSM also suffers from severe conceptual problems indicating that it is incomplete. These include the presence of logarithmic infrared divergences in the $\mathcal{O}\left(\alpha_{s}\right)$ corrections to $P$-wave decays to light hadrons and in the relativistic corrections to $S$-wave annihilation [7], and the lack of a general argument

for its validity in higher orders of perturbation theory. While the $k_{T}$-factorization [8] and hard-comover-scattering [9] approaches manage to bring the CSM prediction much closer to the Tevatron data, they do not cure the conceptual defects of the CSM. The color evaporation model [10], which is intuitive and useful for qualitative studies, also significantly improves the description of the Tevatron data as compared to the CSM [11]. However, it does not account for the process-specific weights of the CS and CO contributions, but rather assumes a fixed ratio of $1: 7$. In this sense, a coequal alternative to the NRQCD factorization formalism is presently not available.

In order to convincingly establish the phenomenological significance of the CO processes, it is indispensable to identify them in other kinds of high-energy experiments as well. Studies of charmonium production in $e p$ photoproduction, ep and $\nu N$ deep-inelastic scattering (DIS), $e^{+} e^{-}$annihilation, $\gamma \gamma$ collisions, and $b$-hadron decays may be found in the literature; see Refs. [12,13] and references cited therein. Furthermore, the polarization of $\psi^{\prime}$ mesons produced directly [14] and of $J / \psi$ mesons produced promptly $[15,16]$, i.e., either directly or via the feed-down from heavier charmonia, which also provides a 
sensitive probe of $\mathrm{CO}$ processes, was investigated. Until recently, none of these studies was able to prove or disprove the NRQCD factorization hypothesis. However, H1 data of $e p \rightarrow e J / \psi+X$ in DIS at HERA [17] and DELPHI data of $\gamma \gamma \rightarrow J / \psi+X$ at LEP2 [18] now provide first independent evidence for it $[19,20]$.

Recently, we studied the associated production of charmonia and electroweak bosons at present and future colliders, including the Tevatron (Run II), the CERN Large Hadron Collider (LHC), the DESY TeV-Energy Superconducting Linear Accelerator (TESLA), and the DESY electron-proton supercollider THERA, which uses the lepton beam of TESLA and the proton beam of the DESY Hadron-Electron Ring Accelerator (HERA) [21]. Our assessment of the observability of the various processes in the various experiments can be summarized as follows: the processes with a photon will abundantly take place in all considered experiments; the processes with a $Z$ or $W$ boson will produce considerable yields at the hadron colliders, namely, several hundred (ten thousand) events per year at the Tevatron (LHC), while they significantly fall short of the one-event-per-year mark at TESLA and THERA; the processes with a standard model (SM) Higgs boson $(\mathcal{H})$ are predicted to be too rare to be observable in any of the considered experiments. As for $J / \psi+W$ associated production at the Tevatron, the conclusions that had been reached in Ref. [22] concur with ours [21]. The associated production of bottomonia and $Z$ or $W$ bosons at the Tevatron and the LHC was investigated in Ref. [23], with the conclusion that such events represent a challenge at the Tevatron, while they should certainly be observable at the LHC. The CDF Collaboration [24] did not find evidence for $\Upsilon(1 S)+Z$ or $\Upsilon(1 S)+W^{ \pm}$signals in Run I at the Tevatron, but established upper bounds on the respective production cross sections, which are well compatible with the theoretical predictions of Ref. [23]. However, with the expected Run II increase of integrated luminosity and the upgrades of the CDF detector, they expect to achieve a sensitivity sufficient to observe these signals [24]. The cross sections of all partonic processes contributing to the associated production of heavy quarkonia, with ${ }^{2 S+1} L_{J}={ }^{1} S_{0},{ }^{3} S_{1},{ }^{1} P_{1},{ }^{3} P_{J}$ $(J=0,1,2)$, and photons, $Z$ bosons, and $W$ bosons in photon-photon, photon-hadron, and hadron-hadron collisions may be found in Ref. [21].

In this paper, we take the next step and consider the production of bottomonia in association with Higgs bosons concentrating on hadron-hadron collisions. Guided by our previous findings in connection with charmonium [21], we expect such signals to be below the edge of observability in two-photon collisions at TESLA and photon-hadron collisions at THERA. Therefore, we do not include the latter production mechanisms in our present phenomenological analysis. However, the relevant partonic cross sections may be obtained from our analytic results by adjusting overall color factors and coupling constants, as is explained in the Appendix.

In the SM, the total cross section of $p p \rightarrow J / \psi+\mathcal{H}$, with Higgs-boson mass $m_{\mathcal{H}}=$ $115 \mathrm{GeV}$, at the LHC was predicted to be $2.0 \times 10^{-2} \mathrm{fb}$, which corresponds to 4 signal events per year [21]. Taking into account the branching fractions of the $J / \psi$ and $\mathcal{H}$ decays to the detected particles and the acceptance cuts required for background suppression, it becomes apparent that the production rate would need to be increased by a few orders of magnitude before a sound signal could be observed. Since the SM Yukawa couplings are 
proportional to the quark masses, passing from charmonium to bottomonium yields an enhancement by a factor of $m_{b}^{2} / m_{c}^{2} \approx 10$. Moreover, the MEs of bottomonium [13,25] are typically one order of magnitude larger than their counterparts for charmonium [15], with the exception of $\left\langle\mathcal{O}^{\Upsilon(n S)}\left[{ }^{1} S_{0}^{(8)}\right]\right\rangle$ and $\left\langle\mathcal{O}^{\Upsilon(n S)}\left[{ }^{3} P_{J}^{(8)}\right]\right\rangle$, which tend to be comparable to their counterparts for $\psi(n S)$. On the other hand, the dynamical quark-mass dependence acts in the opposite direction. In fact, in the case of the total cross section of $p p \rightarrow J / \psi+\mathcal{H}$, with $m_{\mathcal{H}}=115 \mathrm{GeV}$ at the LHC, replacing $m_{c}$ by $m_{b}$ while keeping the Yukawa coupling and the MEs fixed leads to a decrease by a bit more than one order of magnitude, so that the Yukawa coupling enhancement is slightly overtaken. Detailed calculation reveals that the total cross section of $p p \rightarrow \Upsilon(1 S)+\mathcal{H}$ at the LHC exceeds the one of $p p \rightarrow J / \psi+\mathcal{H}$ by a factor of 3.6 , the result being $7.3 \times 10^{-2} \mathrm{fb}$, which corresponds to 15 signal events per year.

The situation should be considerably more promising in the context of supersymmetric theories, where the Yukawa couplings depend on additional input parameters and may be significantly enhanced relative to the reference values in the SM if nature has chosen these parameters accordingly. In the following, we concentrate on the most popular of such theories, the minimal supersymmetric extension of the SM (MSSM). The Higgs sector of the MSSM consists of a two-Higgs-doublet model of type II and accommodates a quintet of physical Higgs bosons: the neutral $C P$-even $h$ and $H$ bosons, the neutral $C P$-odd $A$ boson, and the charged $H^{ \pm}$-boson pair. At the tree level, the MSSM Higgs sector has two free parameters, which are usually taken to be the mass $m_{A}$ of the $A$ boson and the ratio $\tan \beta=v_{2} / v_{1}$ of the vacuum expectation values of the two Higgs doublets. The masses $m_{h}, m_{H}$, and $m_{H^{ \pm}}$of the $h, H$, and $H^{ \pm}$bosons, respectively, and the mixing angle $\alpha$ that rotates the weak $C P$-even Higgs eigenstates into the mass eigenstates $h$ and $H$ are then functions of $m_{A}$ and $\tan \beta$. As is well known, these functions receive significant electroweak radiative corrections, whose leading terms are of $\mathcal{O}\left(G_{F} m_{t}^{4} / m_{Z}^{2}\right)$, where $G_{F}$ is Fermi's constant, $m_{t}$ is the top-quark mass, and $m_{Z}$ is the $Z$-boson mass, and must not be neglected; for a review, see Ref. [26]. For the sake of our exploratory study, it is sufficient to make a few simplifying assumptions in the treatment of these corrections. Specifically, we neglect subleading effects due to nonzero values of the Higgs-higgsino mass parameter $\mu$ and the trilinear Higgs-sfermion couplings $A_{t}$ and $A_{b}$. The leading radiative corrections can then be parameterized by the quantity

$$
\epsilon=\frac{3 G_{F} m_{t}^{4}}{\pi^{2} \sqrt{2} \sin ^{2} \beta} \ln \left(1+\frac{m_{S}^{2}}{m_{t}^{2}}\right),
$$

where $m_{S}$ is the common squark mass, and we have

$$
\begin{aligned}
m_{h}^{2}= & \frac{1}{2}\left[m_{A}^{2}+m_{Z}^{2}+\epsilon\right. \\
& \left.-\sqrt{\left(m_{A}^{2}+m_{Z}^{2}+\epsilon\right)^{2}-4 m_{A}^{2} m_{Z}^{2} \cos ^{2}(2 \beta)-4 \epsilon\left(m_{A}^{2} \sin ^{2} \beta+m_{Z}^{2} \cos ^{2} \beta\right)}\right], \\
m_{H}^{2}= & m_{A}^{2}+m_{Z}^{2}-m_{h}^{2}+\epsilon,
\end{aligned}
$$




$$
\begin{aligned}
m_{H^{ \pm}}^{2} & =m_{A}^{2}+m_{W}^{2}, \\
\tan (2 \alpha) & =\frac{\left(m_{A}^{2}+m_{Z}^{2}\right) \sin (2 \beta)}{\left(m_{A}^{2}-m_{Z}^{2}\right) \cos (2 \beta)+\epsilon},
\end{aligned}
$$

where $m_{W}$ is the $W$-boson mass. Solving Eq. (5) for $\alpha$, one needs to select the branch with $-\pi / 2<\alpha<0$. When $m_{h}$ and $\tan \beta$ are chosen to be the input parameters, then Eq. (2) can be solved for $m_{A}$, which leads to

$$
m_{A}^{2}=\frac{m_{h}^{2}\left(m_{Z}^{2}-m_{h}^{2}\right)+\epsilon\left(m_{h}^{2}-m_{Z}^{2} \cos ^{2} \beta\right)}{m_{Z}^{2} \cos ^{2}(2 \beta)-m_{h}^{2}+\epsilon \sin ^{2} \beta},
$$

and $\alpha$ can be obtained from Eq. (5) as before. From Eq. (6), we glean that the upper bound on $m_{h}^{2}$ is shifted from its tree-level value, $m_{Z}^{2} \cos ^{2}(2 \beta)$, to

$$
m_{h}^{2}<m_{Z}^{2} \cos ^{2}(2 \beta)+\epsilon \sin ^{2} \beta
$$

Combining Eqs. (2) and (3) and noticing that the radicant in Eq. (2) can be written as $\left[m_{A}^{2}-m_{Z}^{2} \cos (4 \beta)+\epsilon \cos (2 \beta)\right]^{2}+\left[m_{Z}^{2} \sin (4 \beta)-\epsilon \sin (2 \beta)\right]^{2}$, we learn that

$$
m_{H}^{2}>m_{h}^{2}+\left|m_{Z}^{2} \sin (4 \beta)-\epsilon \sin (2 \beta)\right| .
$$

Furthermore, inserting Eq. (7) into Eq. (3), we find that

$$
m_{H}^{2}>m_{A}^{2}+m_{Z}^{2} \sin ^{2}(2 \beta)+\epsilon \cos ^{2} \beta .
$$

In other words, $H$ is always heavier than $h$ and $A$.

If we assume only the first four quark flavors to be active in the colliding hadrons, then the associated production of bottomonium and a charged Higgs boson is suppressed by the smallness of the $\bar{d} u H^{-}$and $\bar{s} c H^{-}$couplings. If we also take bottom to be an active flavor, then the final state must contain an additional top quark, $W$ boson, or charged Higgs boson, which substantially squeeze the available phase space and lead to distinctive signals. For these reasons, we only consider the neutral MSSM Higgs bosons $\Phi=h, H, A$ in the following. The $q \bar{q} \Phi$ couplings $y_{q}^{\Phi}$ emerge by scaling the SM Yukawa couplings with factors $g_{q}^{\Phi}$; i.e.,

$$
y_{q}^{\Phi}=2^{1 / 4} G_{F}^{1 / 2} m_{q} g_{q}^{\Phi} .
$$

The values of $g_{q}^{\Phi}$ are specified in Table 1 . From Table 1, we observe that the values of $g_{b}^{\Phi}$ rise linearly with $\tan \beta$ if $\tan \beta \gg 1$. In order to obtain sufficiently large cross sections, we are particularly interested in light Higgs bosons with strong couplings to the $b$ quark. We thus focus our attention on the $h$ and $A$ bosons, and on the large-tan $\beta$ regime.

This paper is organized as follows. In Sec. 2, we present our analytic results and explain how to evaluate the cross sections of the associated production of bottomonia and Higgs bosons in hadronic collisions. The contributing partonic cross sections are collected in the Appendix. In Sec. 3, we present our numerical results. Our conclusions are summarized in Sec. 4. 
Table 1: Values of $g_{q}^{\Phi}$ in Eq. (10) for generic up and down quarks, $U$ and $D$, respectively.

\begin{tabular}{|c|ccc|}
\hline \hline$q$ & $g_{q}^{h}$ & $g_{q}^{H}$ & $g_{q}^{A}$ \\
\hline$U$ & $\cos \alpha / \sin \beta$ & $\sin \alpha / \sin \beta$ & $\cot \beta$ \\
$D$ & $-\sin \alpha / \cos \beta$ & $\cos \alpha / \cos \beta$ & $\tan \beta$ \\
\hline \hline
\end{tabular}

\section{Analytic results}

In this section, we present our analytic results for the cross sections of the reactions $A B \rightarrow C D$, where $A$ and $B$ are the incoming hadrons, $C$ is a heavy-quarkonium state, with ${ }^{2 S+1} L_{J}={ }^{1} S_{0},{ }^{3} S_{1},{ }^{1} P_{1},{ }^{3} P_{J}(J=0,1,2)$, and $D=h, A$. The results for $D=\mathcal{H}, H$ may be obtained from those for $D=h$ by accordingly replacing $y_{Q}^{h}$ and $m_{h}$. We also list the formulas for the cases where $A$ and/or $B$ are real or quasireal photons, appropriate for two-photon and photon-hadron collisions, respectively.

We work in the fixed-flavor-number scheme; i.e., $A$ and $B$ contain $n_{f}=4$ active quark flavors $q=u, d, s, c$ if $Q=b$ and $n_{f}=3$ active quark flavors $q=u, d, s$ if $Q=c$. As required by parton-model kinematics, we treat the quark flavors $q$ as massless. The quark flavor $Q$, with mass $m_{Q}$, only appears in the final state. The $b \bar{b}$ Fock states contributing at LO in $v$ are $n={ }^{1} S_{0}^{(1)},{ }_{1}^{1} S_{0}^{(8)},{ }^{3} S_{1}^{(8)},{ }^{1} P_{1}^{(8)}$ if $H=\eta_{b}(n S) ; n={ }^{3} S_{1}^{(1)},{ }^{1} S_{0}^{(8)},{ }^{3} S_{1}^{(8)},{ }^{3} P_{J}^{(8)}$ if $H=\Upsilon(n S) ; n={ }^{1} P_{1}^{(1)},{ }^{1} S_{0}^{(8)}$ if $H=h_{b}(n P)$; and $n={ }^{3} P_{J}^{(1)},{ }^{3} S_{1}^{(8)}$ if $H=\chi_{b J}(n P)$, where $J=0,1,2$. Their MEs satisfy the multiplicity relations

$$
\begin{aligned}
\left\langle\mathcal{O}^{\Upsilon(n S)}\left[{ }^{3} P_{J}^{(8)}\right]\right\rangle & =(2 J+1)\left\langle\mathcal{O}^{\Upsilon(n S)}\left[{ }^{3} P_{0}^{(8)}\right]\right\rangle, \\
\left\langle\mathcal{O}^{\chi_{b J}(n P)}\left[{ }^{3} P_{J}^{(1)}\right]\right\rangle & =(2 J+1)\left\langle\mathcal{O}^{\chi_{b 0}(n P)}\left[{ }^{3} P_{0}^{(1)}\right]\right\rangle, \\
\left\langle\mathcal{O}^{\chi_{b J}(n P)}\left[{ }^{3} S_{1}^{(8)}\right]\right\rangle & =(2 J+1)\left\langle\mathcal{O}^{\chi_{b 0}(n P)}\left[{ }^{3} S_{1}^{(8)}\right]\right\rangle,
\end{aligned}
$$

which follow to LO in $v$ from heavy-quark spin symmetry. The CS MEs of $\Upsilon(n S)$ and $\chi_{b 0}(n P)$ are related to the respective radial wave functions at the origin as

$$
\begin{aligned}
\left\langle\mathcal{O}^{\Upsilon(n S)}\left[{ }^{3} S_{1}^{(1)}\right]\right\rangle & =\frac{9}{2 \pi}\left|R_{n S}(0)\right|^{2}, \\
\left\langle\mathcal{O}^{\chi_{b 0}(n P)}\left[{ }^{3} P_{0}^{(1)}\right]\right\rangle & =\frac{9}{2 \pi}\left|R_{n P}^{\prime}(0)\right|^{2},
\end{aligned}
$$

respectively. In our numerical analysis, we only include the $\Upsilon(1 S)$ and $\chi_{b J}(1 P)$ mesons. For completeness and future use, we also list formulas for all the other bottomonia. The assignments for the various charmonia are analogous.

We now turn to the partonic subprocesses $a b \rightarrow Q \bar{Q}[n] D$. The differential cross section of such a process is calculated from the pertaining transition-matrix element $\mathcal{T}$ as $d \sigma / d t=\overline{|\mathcal{T}|^{2}} /\left(16 \pi s^{2}\right)$, where the average is over the spin and color degrees of freedom of $a$ and $b$ and the spin of $D$ is summed over. We apply the covariant-projector method 
of Ref. [27] to implement the $Q \bar{Q}$ Fock states $n$ according to the NRQCD factorization formalism.

The following partonic subprocesses contribute to LO in $\alpha_{s}$ and $v$ :

$$
\begin{aligned}
& g g \rightarrow Q \bar{Q}\left[\varsigma^{(1)}\right] D, \\
& g g \rightarrow Q \bar{Q}\left[\varsigma^{(8)}\right] D, \\
& q \bar{q} \rightarrow Q \bar{Q}\left[\varsigma^{(8)}\right] D, \\
& \gamma g \rightarrow Q \bar{Q}\left[\varsigma^{(8)}\right] D, \\
& \gamma \gamma \rightarrow Q \bar{Q}\left[\varsigma^{(1)}\right] D,
\end{aligned}
$$

where $\varsigma={ }^{1} S_{0},{ }^{3} S_{1},{ }^{1} P_{1},{ }^{3} P_{J}$ with $J=0,1,2$. For the reason explained above, $q=c$ must not be included if $Q=c$. The processes $\gamma g \rightarrow Q \bar{Q}\left[\varsigma^{(1)}\right] D$ and $\gamma \gamma \rightarrow Q \bar{Q}\left[\varsigma^{(8)}\right] D$ are forbidden by color conservation. Furthermore, the processes $q \bar{q} \rightarrow Q \bar{Q}\left[\varsigma^{(1)}\right] D$ are prohibited because the $Q$-quark line is connected with the $q$-quark line by one gluon, which transmits color to the $Q \bar{Q}$ pair. Finally, due to charge-conjugation invariance, the processes $g g \rightarrow Q \bar{Q}\left[\varsigma^{(1)}\right] D$, $\gamma g \rightarrow Q \bar{Q}\left[\varsigma^{(8)}\right] D$, and $\gamma \gamma \rightarrow Q \bar{Q}\left[\varsigma^{(1)}\right] D$ are forbidden for $\varsigma={ }^{3} S_{1},{ }^{1} P_{1}$, and the processes $q \bar{q} \rightarrow Q \bar{Q}\left[\varsigma^{(8)}\right] D$ are forbidden for $\varsigma={ }^{1} S_{0},{ }^{3} P_{J}$.

The differential cross sections $d \sigma / d t$ of processes (13)-(17) are listed in the Appendix. We combine the results proportional to the CO MEs $\left\langle\mathcal{O}^{\Upsilon(n S)}\left[{ }^{3} P_{J}^{(8)}\right]\right\rangle$ and $\left\langle\mathcal{O}^{\chi \chi_{b J}(n P)}\left[{ }^{3} S_{1}^{(8)}\right]\right\rangle$, for fixed value of the principal quantum number $n$, exploiting the multiplicity relations of Eq. (11). The results for processes (16) and (17) are obtained from those for processes (13) by adjusting overall color factors and coupling constants as explained in the Appendix. Similar relations also exist between processes (13) and (14) for $\varsigma={ }^{1} S_{0},{ }^{3} P_{J}$.

The evaluation of the hadronic cross sections and their distributions proceeds as explained in Ref. [21]. Specifically, we consider the distributions in the transverse momentum $p_{T}$ common to $C$ and $D$, the rapidities $y_{C}$ and $y_{D}$ of $C$ and $D$, respectively, and the $C D$ invariant mass $m_{C D}$.

\section{$3 \quad$ Numerical results}

We are now in a position to explore the phenomenological consequences of our calculations. We focus our attention on the cases $C=\Upsilon(1 S), \chi_{b J}(1 P)$. These bottomonia can be efficiently identified experimentally, and their MEs are relatively well constrained [13,25]. The predicted cross-section distributions for the $\Upsilon(2 S)$ and $\Upsilon(3 S)$ mesons are similar to those for the $\Upsilon(1 S)$ meson, but their normalizations are somewhat suppressed due to smaller MEs $[13,25]$. The MEs of the $\chi_{b J}(2 P)$ mesons are very similar to those of the $\chi_{b J}(1 P)$ mesons $[13,25]$, and so are their predicted cross-section distributions. The $\eta_{b}(1 S)$ meson needs experimental confirmation, while no events for $\eta_{b}(n S)$ mesons with $n>1$ and $h_{b}(n S)$ mesons have been seen so far [28].

We first describe our theoretical input and the kinematic conditions. We use $m_{b}=$ $4.88 \mathrm{GeV}[13,25], m_{t}=174.3 \mathrm{GeV}, m_{Z}=91.1876 \mathrm{GeV}, G_{F}=1.16639 \times 10^{-5} \mathrm{GeV}^{-2}$, 
and the LO formula for $\alpha_{s}^{\left(n_{f}\right)}\left(\mu_{r}\right)$ [28] with $n_{f}=4$ and asymptotic scale parameter $\Lambda_{\mathrm{QCD}}^{(4)}=192 \mathrm{MeV}[29]$. As for the proton PDFs, we use the LO set from the CTEQ Collaboration (CTEQ5L) [29]. Unless otherwise specified, we choose the renormalization and factorization scales to be $\mu_{r}=\mu_{f}=\sqrt{m_{T}^{C} m_{T}^{D}}$, where $m_{T}^{C}=\sqrt{m_{C}^{2}+p_{T}^{2}}$ is the transverse mass of $C$ and similarly for $D$. We evaluate $\left\langle\mathcal{O}^{\Upsilon(1 S)}\left[{ }^{3} S_{1}^{(1)}\right]\right\rangle$ and $\left\langle\mathcal{O}^{\chi}{ }^{(1)}(1 P)\left[{ }^{3} P_{0}^{(1)}\right]\right\rangle$ from Eq. (12) with the values of $\left|R_{1 S}(0)\right|^{2}$ and $\left|R_{1 P}^{\prime}(0)\right|^{2}$ determined in Ref. [25] using the QCD-motivated potential by Buchmüller and Tye [30]. As for the $\Upsilon(1 S)$ and $\chi_{b J}(1 P) \mathrm{CO}$ MEs, we adopt as our default set the one determined in Ref. [13] through a fit to recent CDF data [31] using the same proton PDFs and CS MEs as we do. Our MSSM benchmark scenario is defined by the parameter set $\tan \beta=50, m_{h}=100 \mathrm{GeV}$ or $m_{A}=100 \mathrm{GeV}$, and $m_{S}=1 \mathrm{TeV}$, and is well inside the currently allowed region of the MSSM parameter space [28]. We then vary $\tan \beta, m_{h}$, and $m_{A}$ one at a time in the ranges $2<\tan \beta<60$, $90<m_{h}<128 \mathrm{GeV}$, and $90<m_{A}<500 \mathrm{GeV}$, respectively. If $D=h$, then we take $m_{h}$ as an input parameter and evaluate $m_{A}$ from Eq. (6). The hadronic center-of-mass energy is $\sqrt{S}=2 \mathrm{TeV}$ in Run II at the Tevatron and $\sqrt{S}=14 \mathrm{TeV}$ at the LHC.

Since our study is at an exploratory level, we refrain from presenting a full-fledged quantitative estimate of the theoretical uncertainties in our predictions. Experience from previous analyses of charmonium production within the NRQCD factorization formalism $[19,20,32]$ leads us to expect relative errors of the order of $\pm 50 \%$. This error estimate should be on the conservative side, since the convergence property of the NRQCD perturbative expansion is expected to be considerably improved as one passes from charmonium to bottomonium, thanks to the reduction in size of $\alpha_{s}$ and $v$. To be specific, we assess the theoretical uncertainties arising from the lack of knowledge of the precise values of the bottomonium MEs and from the freedom in the choice of the renormalization and factorization scales.

We are now in a position to present our numerical results. Figures 1 and 2 are devoted to $p p \rightarrow C D+X$ at the LHC and to $p \bar{p} \rightarrow C D+X$ in Run II at the Tevatron, respectively. In each figure, part (a) gives the $p_{T}$ distributions $d \sigma / d p_{T}$ (upper panel) and the $m_{C D}$ distributions $d \sigma / d m_{C D}$ (lower panel), part (b) the $y_{C}$ distributions $d \sigma / d y_{C}$ (upper panel) and the $y_{D}$ distributions $d \sigma / d y_{D}$ (lower panel), and part (c) the total cross sections $\sigma$ as functions of $\tan \beta$ (upper panel) and $m_{D}$ (lower panel). In each part, the left column refers to $D=h$ and the right one to $D=A$. In each frame, we separately consider $C=\Upsilon(1 S), \chi_{c J}(1 P)$, both in NRQCD and the CSM. It is summed over $C=\chi_{c 0}(1 P), \chi_{c 1}(1 P), \chi_{c 2}(1 P)$. In the following, $n=1$ is implied whenever the label $(n L)$ is omitted.

As is evident from Sec. 2, $\Upsilon+h$ and $\Upsilon+A$ associated production proceeds through process (14) with $\varsigma={ }^{1} S_{0},{ }^{3} S_{1},{ }^{3} P_{J}$ and process (15) with $\varsigma={ }^{3} S_{1}$ (see solid lines in Figs. 1 and 2) and is forbidden to LO in the CSM. On the other hand, $\chi_{b J}+h$ and $\chi_{b J}+A$ associated production is already possible in the CSM, through process (13) with $\varsigma={ }^{3} P_{J}$ (see dotted lines in Figs. 1 and 2); in NRQCD, also the CO processes (14) and (15) with $\varsigma={ }^{3} S_{1}$ contribute.

In the study of the associated production of heavy quarkonia and electroweak gauge 
bosons [21], dominant contributions at $p_{T} \gg m_{C}$ were found to generally arise from so-called fragmentation-prone partonic subprocesses, which contain a gluon with small virtuality, $q^{2}=m_{C}^{2}$, that splits into a $Q \bar{Q}$ pair in the Fock state $n={ }^{3} S_{1}^{(8)}$. Such processes are absent here because the Higgs bosons are always radiated from the outgoing $Q$ or $\bar{Q}$ quark lines before the latter form an asymptotic Fock state $n$.

We now turn to the predictions for the LHC. From Figs. 1(a)-(c), we observe that there is hardly any difference between the various distributions for $D=h$ and their counterparts for $D=A$, except for a trivial difference in the $m_{h}$ and $m_{A}$ dependences, which is due to the upper bound on $m_{h}$, at $m_{h} \approx 128 \mathrm{GeV}$. In each frame, the three curves exhibit similar shapes (except for the $p_{T}$ distributions), but significantly differ in their overall normalizations. Within NRQCD, the results for $C=\chi_{b J}$ typically exceed those for $C=\Upsilon$ by a factor of 2 . As for $C=\chi_{b J}$, the CSM predictions fall short of the NRQCD ones by a factor of 3 to 4 . From Fig. 1(a), we read off that the $p_{T}$ and $m_{C D}$ distributions peak at $p_{T} \approx 6 \mathrm{GeV}$ and $m_{C D} \approx 116 \mathrm{GeV}$, respectively. Beyond their peaks, the CSM predictions for $C=\chi_{b J}$ fall off more rapidly with increasing value of $p_{T}$ than the NRQCD ones. The $y_{C}$ and $y_{D}$ distributions in Fig. 1(b) are symmetric about the origin, reflecting the symmetry of the experimental set-up, and their maxima are rather broad. Figure 1(c) displays the quadratic $\tan \beta$ dependence discussed in Sec. 1 , which is exact for $D=A$, but only approximate for $D=h$. For $m_{h} \lesssim 120 \mathrm{GeV}$, the $m_{h}$ dependence is approximately exponential, following the heuristic law $\sigma(p p \rightarrow C h+X) \propto \exp \left[-4\left(m_{h} / 100 \mathrm{GeV}\right)\right]$. On the other hand, the $m_{A}$ dependence is approximately power-like over the range of values considered, a heuristic law being $\sigma(p p \rightarrow C A+X) \propto m_{A}^{-5}$.

We now move on to the predictions for Run II at the Tevatron. Comparing Figs. 2(a)(c) with Figs. 1(a)-(c), we observe that all distributions are approximately scaled down by a factor of 50 as we pass from the LHC to the Tevatron. Apart from that, their qualitative features essentially remain the same.

Table 2: Total cross sections $\sigma$ in fb of $p p \rightarrow C D+X$ at the LHC and of $p \bar{p} \rightarrow C D+X$ in Run II at the Tevatron, where $C=\Upsilon(1 S), \chi_{b J}(1 P)$ and $D=h, A$, for $\tan \beta=50$ and $m_{D}=100 \mathrm{GeV}$. The values enclosed in parentheses refer to the CSM.

\begin{tabular}{|c|cc|}
\hline \hline$C+D$ & LHC & Tevatron \\
\hline$\Upsilon+h$ & $3.3 \times 10^{2}$ & 6.0 \\
$\chi_{b J}+h$ & $7.4 \times 10^{2}\left(1.9 \times 10^{2}\right)$ & $1.3 \times 10^{1}(3.6)$ \\
$\Upsilon+A$ & $3.4 \times 10^{2}$ & 6.1 \\
$\chi_{b J}+A$ & $7.4 \times 10^{2}\left(1.9 \times 10^{2}\right)$ & $1.3 \times 10^{1}(3.6)$ \\
\hline \hline
\end{tabular}

We now assess the observability of the various processes at the LHC and in Run II the Tevatron. To this end, we list their total cross sections for our MSSM benchmark scenario in Table 2. These values can be converted into annual yields by recalling from Table I of Ref. [21] that a cross section of $0.005 \mathrm{fb}(0.25 \mathrm{fb})$ corresponds to one event 
per year at the LHC (Tevatron). At hadron colliders, the $\Upsilon(1 S)$ mesons are easily detected through their decays to $e^{+} e^{-}$and $\mu^{+} \mu^{-}$pairs, with a combined branching fraction of $B\left(\Upsilon(1 S) \rightarrow l^{+} l^{-}\right)=4.86 \pm 0.13$ [28]. The $\chi_{b J}(1 P)$ mesons radiatively decay to $\Upsilon(1 S)$ mesons, the individual branching fractions being $B\left(\chi_{b 0}(1 P) \rightarrow \Upsilon(1 S) \gamma\right)<6 \%$, $B\left(\chi_{b 1}(1 P) \rightarrow \Upsilon(1 S) \gamma\right)=(35 \pm 8) \%$, and $B\left(\chi_{b 2}(1 P) \rightarrow \Upsilon(1 S) \gamma\right)=(22 \pm 4) \%$ [28]. In the high-tan $\beta$ regime, light $h$ and $A$ bosons predominantly decay to $b \bar{b}$ and $\tau^{+} \tau^{-}$pairs, with branching fractions of about $90 \%$ and $8 \%$, respectively [28]. The $b$ hadrons can be detected by looking for displaced decay vertices with dedicated vertex detectors, even at the LHC. The corresponding numbers of signal events per year estimated assuming detection efficiencies of $100 \%$ are listed in Table 3.

Table 3: Annual numbers of events of $p p \rightarrow C D+X$ at the LHC and of $p \bar{p} \rightarrow C D+X$ in Run II at the Tevatron, where $C=\Upsilon(1 S), \chi_{b J}(1 P)$ and $D=h, A$, for $\tan \beta=50$ and $m_{D}=100 \mathrm{GeV}$. The final-state particles are assumed to be detected via their decays $\chi_{b J}(1 P) \rightarrow \Upsilon(1 S) \gamma, \Upsilon(1 S) \rightarrow e^{+} e^{-}, \mu^{+} \mu^{-}$, and $h, A \rightarrow b \bar{b}, \tau^{+} \tau^{-}$with efficiencies of $100 \%$. The values enclosed in parentheses refer to the CSM.

\begin{tabular}{|c|cc|}
\hline \hline$C+D$ & LHC & Tevatron \\
\hline$\Upsilon+h$ & $3.1 \times 10^{3}$ & 1.1 \\
$\chi_{b J}+h$ & $1.7 \times 10^{3}\left(4.4 \times 10^{2}\right)$ & $6.2 \times 10^{-1}\left(1.6 \times 10^{-1}\right)$ \\
$\Upsilon+A$ & $3.2 \times 10^{3}$ & 1.2 \\
$\chi_{b J}+A$ & $1.7 \times 10^{3}\left(4.5 \times 10^{2}\right)$ & $6.2 \times 10^{-1}\left(1.6 \times 10^{-1}\right)$ \\
\hline \hline
\end{tabular}

We conclude this section by assessing the theoretical uncertainties arising from the lack of knowledge of the precise values of the bottomonium MEs and from the freedom in the choice of the renormalization and factorization scales. Besides the QCD-motivated potential [30], the authors of Ref. [25] also employed three other phenomenological ansätze, namely, a power-law potential [33], a logarithmic potential [34], and a Coulomb-pluslinear potential (Cornell potential) [35]. Taking as the theoretical errors the maximum upward and downward deviations with respect to the results obtained with the QCDmotivated potential, we have $\left\langle\mathcal{O}^{\Upsilon(1 S)}\left[{ }^{3} S_{1}^{(1)}\right]\right\rangle=9.28_{-2.70}^{+10.85} \mathrm{GeV}^{3}$ and $\left\langle\mathcal{O}^{\chi_{b 0}(1 P)}\left[{ }^{3} P_{0}^{(1)}\right]\right\rangle=$ $2.03_{-0.00}^{+0.93} \mathrm{GeV}^{5}$. Since there are no errors assigned to the CO MEs quoted in Ref. [13], for the purpose of this error analysis, we adopt the older results from Ref. [5], which are fitted to earlier CDF data [36]. In compliance with Ref. [13] (see also the discussion in the context of Eq. (4.1) in Ref. [5]), we assume that $\left\langle\mathcal{O}^{\Upsilon(1 S)}\left[{ }^{3} P_{0}^{(8)}\right]\right\rangle=m_{b}^{2}\left\langle\mathcal{O}^{\Upsilon(1 S)}\left[{ }^{1} S_{0}^{(8)}\right]\right\rangle$. We then repeat the evaluation of Table 2 with this set of bottomonium MEs, varying one $\mathrm{ME}$ at a time and combining the individual shifts in quadrature thereby allowing for the upper and lower half-errors to be different. The outcome is presented in Table 4. From Table 4, we observe that the NRQCD results for $C=\Upsilon(1 S), \chi_{b J}(1 P)$ carry errors of $\pm 84 \%$ and $\pm 29 \%$, respectively, while the CSM result for $C=\chi_{b J}(1 P)$ may be increased by up to $47 \%$. We note in passing that CO MEs of Ref. [13] fall outside the error bars of their 
counterparts of Ref. [5], so that the NRQCD results given in Tables 2 and 4 are not actually mutually consistent. While the face values of the NRQCD results in Table 4 might by now be obsolete, we believe that the relative errors should still serve as a useful indicator. The CDF data [36] from which the CO MEs of Ref. [5] were extracted are only based on an integrated luminosity of $16.6 \mathrm{pb}^{-1}$ collected in 1992-1993. The design value of the integrated luminosity to be delivered by the Tevatron until the end of 2009 is currently quoted as $8 \mathrm{fb}^{-1}$ [37]. The combined data sample of the CDF and D0 collaborations would then be three orders of magnitude larger than the one used in Ref. [36], leading to a reduction in statistical error by a factor of about 30 . The experimental errors in the cross section measurements of bottomonium hadroproduction at the Tevatron should then be dominated by systematics.

Table 4: Same as in Table 2, but using the CO MEs of Ref. [5] and taking into account the theoretical errors on the CS [25] and CO [5] MEs.

\begin{tabular}{|c|cc|}
\hline \hline$C+D$ & LHC & Tevatron \\
\hline$\Upsilon+h$ & $(4.4 \pm 3.7) \times 10^{1}$ & $(8.1 \pm 6.8) \times 10^{-1}$ \\
$\chi_{b J}+h$ & $(2.1 \pm 0.6) \times 10^{3}\left(1.9_{-0.0}^{+0.9} \times 10^{2}\right)$ & $(3.8 \pm 1.1) \times 10^{1}\left(3.6_{-0.0}^{+1.7}\right)$ \\
$\Upsilon+A$ & $(4.4 \pm 3.7) \times 10^{1}$ & $(8.2 \pm 6.8) \times 10^{-1}$ \\
$\chi_{b J}+A$ & $(2.1 \pm 0.6) \times 10^{3}\left(1.9_{-0.0}^{+0.9} \times 10^{2}\right)$ & $(3.8 \pm 1.1) \times 10^{1}\left(3.6_{-0.0}^{+1.6}\right)$ \\
\hline \hline
\end{tabular}

Another plausible choice of renormalization and factorization scales is $\mu_{r}=\mu_{f}=m_{D}$. For $p_{T} \ll m_{D}\left(p_{T} \gg m_{D}\right)$, this scale choice is larger (smaller) than our default setting. The $p_{T}$ distributions are particularly sensitive to this change. In order to illustrate this, we recalculated the $p_{T}$ distributions shown in the upper panels of Figs. 1(a) and 2(a) using this alternative scale choice. The results are displayed in the upper and lower panels of Fig. 3, respectively. From Fig. 3, we observe that the $p_{T}$ distributions are appreciably reduced in the lower $p_{T}$ range, for $p_{T} \lesssim 25 \mathrm{GeV}$, as we pass from our default scale choice to the new one. This feature is more pronounced for the Tevatron than for the LHC. On the other hand, towards the upper end of the $p_{T}$ values considered in the case of the LHC, the shifts in cross section induced by switching to the new scale choice are insignificant. Such scale uncertainties are generally expected to be reduced as next-to-leading-order corrections are taken into account.

\section{Conclusions}

We studied the associated production of heavy quarkonia $C$ and Higgs bosons $D$ in photonphoton, photon-hadron, and hadron-hadron collisions to LO in the NRQCD factorization formalism. We considered all experimentally established heavy quarkonia, with ${ }^{2 S+1} L_{J}=$ ${ }^{1} S_{0},{ }^{3} S_{1},{ }^{1} P_{1},{ }^{3} P_{J}(J=0,1,2)$, and the SM Higgs boson $D=\mathcal{H}$ as well as the MSSM 
Higgs bosons $D=h, H, A, H^{ \pm}$. We listed the cross sections of all contributing partonic subprocesses, except for those with $D=H^{ \pm}$, which are either suppressed by small Yukawa couplings or involve an additional heavy particle with a distinct signature in the final state. We presented numerical results for any combination of $C=\Upsilon(1 S), \chi_{b J}(1 P)$ and $D=h, A$ appropriate for the LHC and Run II at the Tevatron, concentrating on the region of the currently allowed MSSM parameter space characterized by a large value of $\tan \beta$ and small values of $m_{h}$ and $m_{A}$.

Criteria to discriminate NRQCD against the CSM include the following: (i) $\Upsilon+h$ and $\Upsilon+A$ associated production is allowed in NRQCD, but forbidden to LO in the CSM; and (ii) in NRQCD, the cross sections of $\chi_{b J}+h$ and $\chi_{b J}+A$ associated production are a factor of 3 to 4 larger than in the CSM.

In our MSSM benchmark scenario, there will be about 3000 (1) direct $\Upsilon(1 S)+h \rightarrow$ $l^{+} l^{-}+b \bar{b}$ events per year at the LHC (Tevatron) and similarly for the $A$ boson. The $\chi_{b J} \rightarrow \Upsilon(1 S) \gamma$ feed-down channels will add about 1500 (0.5) events to each of these yields. Results in the same ballpark are expected for the bottomonia with $n=2,3$. We conclude that these signals should be clearly visible at the LHC, while they should provide a challenge for Run II at the Tevatron.

\section{Acknowledgments}

B.A.K. and C.P.P. are grateful to the Kavli Institute for Theoretical Physics at the University of California at Santa Barbara and the Second Institute for Theoretical Physics at the University of Hamburg, respectively, for their hospitality during visits when this paper was prepared. The research of B.A.K. was supported in part by the Deutsche Forschungsgemeinschaft (DFG) under Grant No. KN 365/1, by the Bundesministerium für Bildung und Forschung under Grant No. 05 HT1GUA/4, and by the National Science Foundation under Grant No. PHY99-07949. The research of C.P.P. was supported in part by the DFG through Graduiertenkolleg No. GRK 602/1 and by the Office of the Vice President for Academic Affairs of the University of the Philippines. The research of L.Z. was supported by the Studienstiftung des deutschen Volkes through a PhD scholarship. 


\section{A Partonic cross sections}

In this appendix, we list the differential cross sections $d \sigma / d t$ for processes (13)-(17) with $D=h, A$. The results for $D=\mathcal{H}, H$ may be obtained from those for $D=h$ by accordingly replacing $y_{Q}^{h}$ and $m_{h}$. Expressions of the partonic Mandelstam variables $s, t$, and $u$ in terms of $p_{T}, y_{C}$, and $y_{D}$ may be found in Eq. (4) of Ref. [21]. The mass $m_{C}=M$ of the heavy quarkonium is taken to be $M=2 m_{Q}$.

$$
\begin{aligned}
& \frac{d \sigma}{d t}\left(g g \rightarrow Q \bar{Q}\left[{ }^{1} S_{0}^{(1)}\right] h\right)=\frac{2 \pi \alpha_{s}^{2}\left(y_{Q}^{h}\right)^{2} M\left(2 m_{h}^{2}-s-t-u\right)^{2}}{9\left(m_{h}^{2}-s-t\right)^{2}\left(m_{h}^{2}-s-u\right)^{2}\left(2 m_{h}^{2}-t-u\right)^{2}}, \\
& \frac{d \sigma}{d t}\left(g g \rightarrow Q \bar{Q}\left[{ }^{3} P_{J}^{(1)}\right] h\right)=\frac{-4 \pi \alpha_{s}^{2}\left(y_{Q}^{h}\right)^{2}}{135 M^{3} s^{2}\left(m_{h}^{2}-s-t\right)^{4}\left(m_{h}^{2}-s-u\right)^{4}\left(2 m_{h}^{2}-t-u\right)^{4}} \\
& \times F_{J}, \quad J=0,1,2, \\
& F_{0}=-10 M^{2}\left\{196 m_{h}^{20}-140 m_{h}^{18}[8 s+7(t+u)]\right. \\
& +m_{h}^{16}\left[2756 s^{2}+4844 s(t+u)+35\left(59 t^{2}+134 t u+59 u^{2}\right)\right] \\
& -2 m_{h}^{14}\left[1920 s^{3}+5092 s^{2}(t+u)+s\left(4329 t^{2}+9934 t u+4329 u^{2}\right)\right. \\
& \left.+70(t+u)\left(17 t^{2}+50 t u+17 u^{2}\right)\right] \\
& +2 m_{h}^{12}\left[1680 s^{4}+6012 s^{3}(t+u)+4 s^{2}\left(1908 t^{2}+4409 t u+1908 u^{2}\right)\right. \\
& +7 s(t+u)\left(589 t^{2}+1790 t u+589 u^{2}\right) \\
& \left.+2\left(407 t^{4}+2537 t^{3} u+4402 t^{2} u^{2}+2537 t u^{3}+407 u^{4}\right)\right] \\
& -2 m_{h}^{10}\left[968 s^{5}+4462 s^{4}(t+u)+s^{3}\left(7559 t^{2}+17434 t u+7559 u^{2}\right)\right. \\
& +2 s^{2}(t+u)\left(2983 t^{2}+9210 t u+2983 u^{2}\right) \\
& +s\left(2245 t^{4}+14659 t^{3} u+25776 t^{2} u^{2}+14659 t u^{3}+2245 u^{4}\right) \\
& \left.+(t+u)\left(335 t^{4}+2874 t^{3} u+5930 t^{2} u^{2}+2874 t u^{3}+335 u^{4}\right)\right] \\
& +m_{h}^{8}\left[740 s^{6}+4412 s^{5}(t+u)+2 s^{4}\left(4803 t^{2}+10882 t u+4803 u^{2}\right)\right. \\
& +2 s^{3}(t+u)\left(4959 t^{2}+14882 t u+4959 u^{2}\right) \\
& +s^{2}\left(5219 t^{4}+34390 t^{3} u+60782 t^{2} u^{2}+34390 t u^{3}+5219 u^{4}\right) \\
& +14 s(t+u)\left(99 t^{4}+934 t^{3} u+1994 t^{2} u^{2}+934 t u^{3}+99 u^{4}\right) \\
& \left.+10\left(16 t^{6}+239 t^{5} u+1007 t^{4} u^{2}+1592 t^{3} u^{3}+1007 t^{2} u^{4}+239 t u^{5}+16 u^{6}\right)\right] \\
& -2 m_{h}^{6}\left[88 s^{7}+728 s^{6}(t+u)+s^{5}\left(2089 t^{2}+4574 t u+2089 u^{2}\right)\right. \\
& +26 s^{4}(t+u)\left(107 t^{2}+292 t u+107 u^{2}\right) \\
& +5 s^{3}\left(375 t^{4}+2270 t^{3} u+3942 t^{2} u^{2}+2270 t u^{3}+375 u^{4}\right) \\
& +s^{2}(t+u)\left(643 t^{4}+5906 t^{3} u+12746 t^{2} u^{2}+5906 t u^{3}+643 u^{4}\right) \\
& +s\left(111 t^{6}+1965 t^{5} u+8844 t^{4} u^{2}+14224 t^{3} u^{3}+8844 t^{2} u^{4}+1965 t u^{5}+111 u^{6}\right) \\
& \left.+10(t+u)\left(t^{2}+5 t u+u^{2}\right)\left(t^{4}+19 t^{3} u+44 t^{2} u^{2}+19 t u^{3}+u^{4}\right)\right] \\
& +m_{h}^{4}\left[20 s^{8}+288 s^{7}(t+u)+8 s^{6}\left(149 t^{2}+314 t u+149 u^{2}\right)\right. \\
& +2 s^{5}(t+u)\left(1077 t^{2}+2578 t u+1077 u^{2}\right)
\end{aligned}
$$




$$
\begin{aligned}
& +2 s^{4}\left(969 t^{4}+4952 t^{3} u+8206 t^{2} u^{2}+4952 t u^{3}+969 u^{4}\right) \\
& +2 s^{3}(t+u)\left(437 t^{4}+3048 t^{3} u+6210 t^{2} u^{2}+3048 t u^{3}+437 u^{4}\right) \\
& +s^{2}\left(179 t^{6}+2630 t^{5} u+11681 t^{4} u^{2}+18788 t^{3} u^{3}+11681 t^{2} u^{4}+2630 t u^{5}+179 u^{6}\right) \\
& +2 s(t+u)\left(7 t^{6}+258 t^{5} u+1740 t^{4} u^{2}+3326 t^{3} u^{3}+1740 t^{2} u^{4}+258 t u^{5}+7 u^{6}\right) \\
& \left.+t^{8}+52 t^{7} u+568 t^{6} u^{2}+2144 t^{5} u^{3}+3290 t^{4} u^{4}+2144 t^{3} u^{5}+568 t^{2} u^{6}+52 t u^{7}+u^{8}\right] \\
& -2 m_{h}^{2}\left[12 s^{8}(t+u)+s^{7}\left(91 t^{2}+186 t u+91 u^{2}\right)+2 s^{6}(t+u)\left(121 t^{2}+260 t u+121 u^{2}\right)\right. \\
& +s^{5}\left(308 t^{4}+1361 t^{3} u+2142 t^{2} u^{2}+1361 t u^{3}+308 u^{4}\right) \\
& +2 s^{4}(t+u)\left(101 t^{4}+511 t^{3} u+919 t^{2} u^{2}+511 t u^{3}+101 u^{4}\right) \\
& +s^{3}\left(65 t^{6}+566 t^{5} u+2070 t^{4} u^{2}+3182 t^{3} u^{3}+2070 t^{2} u^{4}+566 t u^{5}+65 u^{6}\right) \\
& +s^{2}(t+u)\left(8 t^{6}+119 t^{5} u+744 t^{4} u^{2}+1418 t^{3} u^{3}+744 t^{2} u^{4}+119 t u^{5}+8 u^{6}\right) \\
& +\operatorname{stu}\left(13 t^{6}+204 t^{5} u+852 t^{4} u^{2}+1334 t^{3} u^{3}+852 t^{2} u^{4}+204 t u^{5}+13 u^{6}\right) \\
& \left.+t u(t+u)\left(t^{2}+5 t u+u^{2}\right)\left(t^{4}+16 t^{3} u+36 t^{2} u^{2}+16 t u^{3}+u^{4}\right)\right] \\
& +9 s^{8}(t+u)^{2}+42 s^{7}(t+u)^{3}+s^{6}\left(79 t^{4}+322 t^{3} u+490 t^{2} u^{2}+322 t u^{3}+79 u^{4}\right) \\
& +4 s^{5}(t+u)\left(19 t^{4}+81 t^{3} u+131 t^{2} u^{2}+81 t u^{3}+19 u^{4}\right) \\
& +s^{4}\left(39 t^{6}+258 t^{5} u+755 t^{4} u^{2}+1080 t^{3} u^{3}+755 t^{2} u^{4}+258 t u^{5}+39 u^{6}\right) \\
& +2 s^{3}(t+u)\left(5 t^{6}+36 t^{5} u+144 t^{4} u^{2}+246 t^{3} u^{3}+144 t^{2} u^{4}+36 t u^{5}+5 u^{6}\right) \\
& +s^{2}\left(t^{8}+10 t^{7} u+91 t^{6} u^{2}+356 t^{5} u^{3}+552 t^{4} u^{4}+356 t^{3} u^{5}+91 t^{2} u^{6}+10 t u^{7}+u^{8}\right) \\
& +4 s t^{2} u^{2}(t+u)\left(t^{2}+5 t u+u^{2}\right)\left(3 t^{2}+7 t u+3 u^{2}\right) \\
& \left.+t^{2} u^{2}(t+u)^{2}\left(t^{2}+5 t u+u^{2}\right)^{2}\right\} \text {, } \\
& F_{1}=-5\left(2 m_{h}^{2}-s-t-u\right)^{2}\left\{4 m_{h}^{16} s-4 m_{h}^{14}\left[10 s^{2}+4 s(t+u)+(t-u)^{2}\right]\right. \\
& +2 m_{h}^{12}\left[62 s^{3}+72 s^{2}(t+u)+s\left(23 t^{2}+10 t u+23 u^{2}\right)+8(t+u)(t-u)^{2}\right] \\
& -m_{h}^{10}\left[176 s^{4}+368 s^{3}(t+u)+2 s^{2}\left(119 t^{2}+206 t u+119 u^{2}\right)\right. \\
& \left.+2 s(t+u)\left(47 t^{2}-38 t u+47 u^{2}\right)+(t-u)^{2}\left(25 t^{2}+58 t u+25 u^{2}\right)\right] \\
& +m_{h}^{8}\left[124 s^{5}+400 s^{4}(t+u)+4 s^{3}\left(109 t^{2}+234 t u+109 u^{2}\right)\right. \\
& +4 s^{2}(t+u)\left(61 t^{2}+68 t u+61 u^{2}\right)+s\left(113 t^{4}+86 t^{3} u-118 t^{2} u^{2}+86 t u^{3}+113 u^{4}\right) \\
& \left.+(t+u)(t-u)^{2}\left(19 t^{2}+62 t u+19 u^{2}\right)\right] \\
& -m_{h}^{6}\left[40 s^{6}+192 s^{5}(t+u)+8 s^{4}\left(41 t^{2}+96 t u+41 u^{2}\right)\right. \\
& +8 s^{3}(t+u)\left(34 t^{2}+79 t u+34 u^{2}\right) \\
& +2 s^{2}\left(83 t^{4}+194 t^{3} u+226 t^{2} u^{2}+194 t u^{3}+83 u^{4}\right) \\
& +4 s(t+u)\left(19 t^{4}+17 t^{3} u-44 t^{2} u^{2}+17 t u^{3}+19 u^{4}\right) \\
& \left.+(t-u)^{2}\left(7 t^{4}+54 t^{3} u+98 t^{2} u^{2}+54 t u^{3}+7 u^{4}\right)\right] \\
& +m_{h}^{4}\left[4 s^{7}+32 s^{6}(t+u)+2 s^{5}\left(47 t^{2}+114 t u+47 u^{2}\right)\right. \\
& +4 s^{4}(t+u)\left(31 t^{2}+92 t u+31 u^{2}\right) \\
& +2 s^{3}\left(51 t^{4}+206 t^{3} u+340 t^{2} u^{2}+206 t u^{3}+51 u^{4}\right)+10 s^{2}(t+u)^{3}\left(7 t^{2}-2 t u+7 u^{2}\right) \\
& +s\left(27 t^{6}+100 t^{5} u-t^{4} u^{2}-140 t^{3} u^{3}-t^{2} u^{4}+100 t u^{5}+27 u^{6}\right) \\
& \left.+(t+u)(t-u)^{2}\left(t^{4}+16 t^{3} u+38 t^{2} u^{2}+16 t u^{3}+u^{4}\right)\right]
\end{aligned}
$$




$$
\begin{aligned}
& -m_{h}^{2}\left[6 s^{6}(t+u)^{2}+2 s^{5}(t+u)\left(9 t^{2}+26 t u+9 u^{2}\right)\right. \\
& +s^{4}\left(25 t^{4}+124 t^{3} u+214 t^{2} u^{2}+124 t u^{3}+25 u^{4}\right) \\
& +4 s^{3}(t+u)\left(6 t^{4}+17 t^{3} u+36 t^{2} u^{2}+17 t u^{3}+6 u^{4}\right) \\
& +s^{2}\left(15 t^{6}+56 t^{5} u+63 t^{4} u^{2}+60 t^{3} u^{3}+63 t^{2} u^{4}+56 t u^{5}+15 u^{6}\right) \\
& +2 s(t+u)\left(2 t^{6}+14 t^{5} u-3 t^{4} u^{2}-18 t^{3} u^{3}-3 t^{2} u^{4}+14 t u^{5}+2 u^{6}\right) \\
& \left.+t u\left(t^{2}-u^{2}\right)^{2}\left(2 t^{2}+9 t u+2 u^{2}\right)\right] \\
& +s^{5}\left(t^{4}+6 t^{3} u+6 t^{2} u^{2}+6 t u^{3}+u^{4}\right) \\
& +s^{4}(t+u)\left(3 t^{4}+8 t^{3} u+18 t^{2} u^{2}+8 t u^{3}+3 u^{4}\right) \\
& +s^{3}\left(3 t^{6}+8 t^{5} u+25 t^{4} u^{2}+32 t^{3} u^{3}+25 t^{2} u^{4}+8 t u^{5}+3 u^{6}\right) \\
& +s^{2}(t+u)\left(t^{6}+6 t^{5} u+3 t^{4} u^{2}+4 t^{3} u^{3}+3 t^{2} u^{4}+6 t u^{5}+u^{6}\right) \\
& +\operatorname{stu}\left(4 t^{6}+5 t^{5} u-2 t^{4} u^{2}-10 t^{3} u^{3}-2 t^{2} u^{4}+5 t u^{5}+4 u^{6}\right) \\
& \left.+t^{2} u^{2}(t-u)^{2}(t+u)^{3}\right\} \\
& F_{2}=448 m_{h}^{22}-16 m_{h}^{20}[149 s+168(t+u)] \\
& +16 m_{h}^{18}\left[354 s^{2}+800 s(t+u)+7\left(63 t^{2}+134 t u+63 u^{2}\right)\right] \\
& -4 m_{h}^{16}\left[2111 s^{3}+6834 s^{2}(t+u)+s\left(7491 t^{2}+15766 t u+7491 u^{2}\right)\right. \\
& \left.+140(t+u)\left(19 t^{2}+46 t u+19 u^{2}\right)\right] \\
& +4 m_{h}^{14}\left[2358 s^{4}+9272 s^{3}(t+u)+s^{2}\left(14369 t^{2}+29782 t u+14369 u^{2}\right)\right. \\
& +s(t+u)\left(10041 t^{2}+23470 t u+10041 u^{2}\right) \\
& \left.+7\left(363 t^{4}+1796 t^{3} u+2882 t^{2} u^{2}+1796 t u^{3}+363 u^{4}\right)\right] \\
& -2 m_{h}^{12}\left[4130 s^{5}+18820 s^{4}(t+u)+5 s^{3}\left(7093 t^{2}+14430 t u+7093 u^{2}\right)\right. \\
& +26 s^{2}(t+u)\left(1329 t^{2}+2954 t u+1329 u^{2}\right) \\
& +s\left(16995 t^{4}+80520 t^{3} u+127418 t^{2} u^{2}+80520 t u^{3}+16995 u^{4}\right) \\
& \left.+56(t+u)\left(57 t^{4}+332 t^{3} u+566 t^{2} u^{2}+332 t u^{3}+57 u^{4}\right)\right] \\
& +m_{h}^{10}\left[5232 s^{6}+28832 s^{5}(t+u)+2 s^{4}\left(32427 t^{2}+64870 t u+32427 u^{2}\right)\right. \\
& +2 s^{3}(t+u)\left(38653 t^{2}+80838 t u+38653 u^{2}\right) \\
& +s^{2}\left(52313 t^{4}+233504 t^{3} u+362542 t^{2} u^{2}+233504 t u^{3}+52313 u^{4}\right) \\
& +4 s(t+u)\left(4710 t^{4}+25333 t^{3} u+41834 t^{2} u^{2}+25333 t u^{3}+4710 u^{4}\right) \\
& \left.+7(t+u)^{2}\left(377 t^{4}+2748 t^{3} u+5174 t^{2} u^{2}+2748 t u^{3}+377 u^{4}\right)\right] \\
& -m_{h}^{8}\left[2116 s^{7}+15080 s^{6}(t+u)+224 s^{5}\left(191 t^{2}+377 t u+191 u^{2}\right)\right. \\
& +4 s^{4}(t+u)\left(15689 t^{2}+31000 t u+15689 u^{2}\right) \\
& +s^{3}\left(52571 t^{4}+218938 t^{3} u+332542 t^{2} u^{2}+218938 t u^{3}+52571 u^{4}\right) \\
& +s^{2}(t+u)\left(25783 t^{4}+124384 t^{3} u+197458 t^{2} u^{2}+124384 t u^{3}+25783 u^{4}\right) \\
& +s\left(6841 t^{6}+57484 t^{5} u+171535 t^{4} u^{2}+241720 t^{3} u^{3}\right. \\
& \left.+171535 t^{2} u^{4}+57484 t u^{5}+6841 u^{6}\right) \\
& \left.+7(t+u)^{3}\left(99 t^{4}+980 t^{3} u+2162 t^{2} u^{2}+980 t u^{3}+99 u^{4}\right)\right] \\
& +m_{h}^{6}\left[472 s^{8}+4752 s^{7}(t+u)+8 s^{6}\left(2279 t^{2}+4436 t u+2279 u^{2}\right)\right.
\end{aligned}
$$




$$
\begin{aligned}
& +4 s^{5}(t+u)\left(8689 t^{2}+16336 t u+8689 u^{2}\right) \\
& +2 s^{4}\left(18433 t^{4}+72142 t^{3} u+107398 t^{2} u^{2}+72142 t u^{3}+18433 u^{4}\right) \\
& +4 s^{3}(t+u)\left(5706 t^{4}+24309 t^{3} u+37018 t^{2} u^{2}+24309 t u^{3}+5706 u^{4}\right) \\
& +s^{2}\left(8227 t^{6}+60592 t^{5} u+168433 t^{4} u^{2}+232088 t^{3} u^{3}\right. \\
& \left.+168433 t^{2} u^{4}+60592 t u^{5}+8227 u^{6}\right) \\
& +2 s(t+u)\left(785 t^{6}+7956 t^{5} u+25901 t^{4} u^{2}+37404 t^{3} u^{3}\right. \\
& \left.+25901 t^{2} u^{4}+7956 t u^{5}+785 u^{6}\right) \\
& \left.+35(t+u)^{4}\left(3 t^{4}+46 t^{3} u+130 t^{2} u^{2}+46 t u^{3}+3 u^{4}\right)\right] \\
& -m_{h}^{4}\left[44 s^{9}+776 s^{8}(t+u)+2 s^{7}\left(2229 t^{2}+4262 t u+2229 u^{2}\right)\right. \\
& +8 s^{6}(t+u)\left(1453 t^{2}+2593 t u+1453 u^{2}\right) \\
& +4 s^{5}\left(4066 t^{4}+15109 t^{3} u+22095 t^{2} u^{2}+15109 t u^{3}+4066 u^{4}\right) \\
& +2 s^{4}(t+u)\left(6549 t^{4}+25090 t^{3} u+36846 t^{2} u^{2}+25090 t u^{3}+6549 u^{4}\right) \\
& +s^{3}\left(6151 t^{6}+39452 t^{5} u+101439 t^{4} u^{2}+136332 t^{3} u^{3}\right. \\
& \left.+101439 t^{2} u^{4}+39452 t u^{5}+6151 u^{6}\right) \\
& +s^{2}(t+u)\left(1619 t^{6}+13326 t^{5} u+38633 t^{4} u^{2}+53884 t^{3} u^{3}\right. \\
& \left.+38633 t^{2} u^{4}+13326 t u^{5}+1619 u^{6}\right) \\
& +s\left(207 t^{8}+3214 t^{7} u+16050 t^{6} u^{2}+38562 t^{5} u^{3}+50990 t^{4} u^{4}\right. \\
& \left.+38562 t^{3} u^{5}+16050 t^{2} u^{6}+3214 t u^{7}+207 u^{8}\right) \\
& \left.+7(t+u)^{5}\left(t^{4}+32 t^{3} u+134 t^{2} u^{2}+32 t u^{3}+u^{4}\right)\right] \\
& +m_{h}^{2}\left[48 s^{9}(t+u)+2 s^{8}\left(271 t^{2}+510 t u+271 u^{2}\right)\right. \\
& +2 s^{7}(t+u)\left(1023 t^{2}+1726 t u+1023 u^{2}\right) \\
& +s^{6}\left(3849 t^{4}+13644 t^{3} u+19606 t^{2} u^{2}+13644 t u^{3}+3849 u^{4}\right) \\
& +4 s^{5}(t+u)\left(1027 t^{4}+3626 t^{3} u+5160 t^{2} u^{2}+3626 t u^{3}+1027 u^{4}\right) \\
& +2 s^{4}\left(1290 t^{6}+7467 t^{5} u+17981 t^{4} u^{2}+23632 t^{3} u^{3}+17981 t^{2} u^{4}+7467 t u^{5}+1290 u^{6}\right) \\
& +10 s^{3}(t+u)\left(93 t^{6}+626 t^{5} u+1608 t^{4} u^{2}+2162 t^{3} u^{3}+1608 t^{2} u^{4}+626 t u^{5}+93 u^{6}\right) \\
& +s^{2}\left(173 t^{8}+2052 t^{7} u+8737 t^{6} u^{2}+19348 t^{5} u^{3}+24948 t^{4} u^{4}\right. \\
& \left.+19348 t^{3} u^{5}+8737 t^{2} u^{6}+2052 t u^{7}+173 u^{8}\right) \\
& +4 s(t+u)\left(3 t^{8}+76 t^{7} u+441 t^{6} u^{2}+1135 t^{5} u^{3}+1522 t^{4} u^{4}\right. \\
& \left.\left.+1135 t^{3} u^{5}+441 t^{2} u^{6}+76 t u^{7}+3 u^{8}\right)+7 t u(t+u)^{6}\left(2 t^{2}+17 t u+2 u^{2}\right)\right] \\
& -24 s^{9}(t+u)^{2}-48 s^{8}(t+u)\left(3 t^{2}+5 t u+3 u^{2}\right) \\
& -s^{7}\left(367 t^{4}+1258 t^{3} u+1786 t^{2} u^{2}+1258 t u^{3}+367 u^{4}\right) \\
& -s^{6}(t+u)\left(515 t^{4}+1708 t^{3} u+2370 t^{2} u^{2}+1708 t u^{3}+515 u^{4}\right) \\
& -2 s^{5}\left(215 t^{6}+1162 t^{5} u+2665 t^{4} u^{2}+3440 t^{3} u^{3}+2665 t^{2} u^{4}+1162 t u^{5}+215 u^{6}\right) \\
& -2 s^{4}(t+u)\left(107 t^{6}+630 t^{5} u+1477 t^{4} u^{2}+1920 t^{3} u^{3}+1477 t^{2} u^{4}+630 t u^{5}+107 u^{6}\right) \\
& -s^{3}\left(59 t^{8}+558 t^{7} u+2051 t^{6} u^{2}+4160 t^{5} u^{3}+5220 t^{4} u^{4}\right. \\
& \left.+4160 t^{3} u^{5}+2051 t^{2} u^{6}+558 t u^{7}+59 u^{8}\right)
\end{aligned}
$$




$$
\begin{aligned}
& -s^{2}(t+u)\left(7 t^{8}+112 t^{7} u+505 t^{6} u^{2}+1152 t^{5} u^{3}+1496 t^{4} u^{4}\right. \\
& \left.+1152 t^{3} u^{5}+505 t^{2} u^{6}+112 t u^{7}+7 u^{8}\right) \\
& -s t u(t+u)^{2}\left(12 t^{6}+85 t^{5} u+242 t^{4} u^{2}+326 t^{3} u^{3}+242 t^{2} u^{4}+85 t u^{5}+12 u^{6}\right) \\
& -7 t^{2} u^{2}(t+u)^{7} \\
& \frac{d \sigma}{d t}\left(g g \rightarrow Q \bar{Q}\left[{ }^{1} S_{0}^{(8)}\right] h\right)=\frac{15}{8} \frac{d \sigma}{d t}\left(g g \rightarrow Q \bar{Q}\left[{ }^{1} S_{0}^{(1)}\right] h\right), \\
& \frac{d \sigma}{d t}\left(g g \rightarrow Q \bar{Q}\left[{ }^{3} S_{1}^{(8)}\right] h\right)=\frac{\pi \alpha_{s}^{2}\left(y_{Q}^{h}\right)^{2}}{4 M s^{3}\left(m_{h}^{2}-s-t\right)^{2}\left(m_{h}^{2}-s-u\right)^{2}\left(2 m_{h}^{2}-t-u\right)^{2}} \\
& \times\left\{9 m_{h}^{12}-9 m_{h}^{10}[2 s+3(t+u)]+m_{h}^{8}\left[10 s^{2}+40 s(t+u)+3\left(11 t^{2}+23 t u+11 u^{2}\right)\right]\right. \\
& -m_{h}^{6}\left[s^{3}+15 s^{2}(t+u)+4 s\left(9 t^{2}+17 t u+9 u^{2}\right)+3(t+u)\left(7 t^{2}+16 t u+7 u^{2}\right)\right] \\
& +m_{h}^{4}\left[s^{3}(t+u)+2 s^{2}\left(5 t^{2}+6 t u+5 u^{2}\right)+4 s(t+u)\left(4 t^{2}+7 t u+4 u^{2}\right)\right. \\
& \left.+7 t^{4}+35 t^{3} u+51 t^{2} u^{2}+35 t u^{3}+7 u^{4}\right] \\
& -m_{h}^{2}\left[s^{3}\left(t^{2}-t u+u^{2}\right)+s^{2}(t+u)\left(3 t^{2}+t u+3 u^{2}\right)\right. \\
& \left.+s\left(3 t^{4}+14 t^{3} u+16 t^{2} u^{2}+14 t u^{3}+3 u^{4}\right)+(t+u)\left(t^{2}+t u+u^{2}\right)\left(t^{2}+7 t u+u^{2}\right)\right] \\
& \left.+t u\left[s^{2}\left(t^{2}+u^{2}\right)+2 s(t+u)\left(t^{2}+u^{2}\right)+\left(t^{2}+t u+u^{2}\right)^{2}\right]\right\}, \\
& \frac{d \sigma}{d t}\left(g g \rightarrow Q \bar{Q}\left[{ }^{1} P_{1}^{(8)}\right] h\right)=\frac{\pi \alpha_{s}^{2}\left(y_{Q}^{h}\right)^{2}}{M^{3} s^{3}\left(m_{h}^{2}-s-t\right)^{3}\left(m_{h}^{2}-s-u\right)^{3}\left(2 m_{h}^{2}-t-u\right)^{4}} \\
& \times\left\{36 m_{h}^{20}-4 m_{h}^{18}[38 s+45(t+u)]\right. \\
& +m_{h}^{16}\left[300 s^{2}+668 s(t+u)+3\left(131 t^{2}+278 t u+131 u^{2}\right)\right] \\
& -2 m_{h}^{14}\left[182 s^{3}+586 s^{2}(t+u)+s\left(639 t^{2}+1330 t u+639 u^{2}\right)\right. \\
& \left.+6(t+u)\left(41 t^{2}+98 t u+41 u^{2}\right)\right] \\
& +m_{h}^{12}\left[308 s^{4}+1256 s^{3}(t+u)+s^{2}\left(1979 t^{2}+4050 t u+1979 u^{2}\right)\right. \\
& \left.+7 s(t+u)\left(199 t^{2}+450 t u+199 u^{2}\right)+4\left(97 t^{4}+473 t^{3} u+750 t^{2} u^{2}+473 t u^{3}+97 u^{4}\right)\right] \\
& -m_{h}^{10}\left[204 s^{5}+940 s^{4}(t+u)+s^{3}\left(1841 t^{2}+3722 t u+1841 u^{2}\right)\right. \\
& +2 s^{2}(t+u)\left(937 t^{2}+2032 t u+937 u^{2}\right) \\
& +7 s\left(135 t^{4}+622 t^{3} u+966 t^{2} u^{2}+622 t u^{3}+135 u^{4}\right) \\
& \left.+6(t+u)\left(33 t^{4}+190 t^{3} u+310 t^{2} u^{2}+190 t u^{3}+33 u^{4}\right)\right] \\
& +m_{h}^{8}\left[104 s^{6}+552 s^{5}(t+u)+2 s^{4}\left(597 t^{2}+1198 t u+597 u^{2}\right)\right. \\
& +4 s^{3}(t+u)\left(367 t^{2}+776 t u+367 u^{2}\right) \\
& +s^{2}\left(1074 t^{4}+4779 t^{3} u+7334 t^{2} u^{2}+4779 t u^{3}+1074 u^{4}\right) \\
& +s(t+u)\left(407 t^{4}+2145 t^{3} u+3352 t^{2} u^{2}+2145 t u^{3}+407 u^{4}\right) \\
& \left.+2\left(32 t^{6}+303 t^{5} u+915 t^{4} u^{2}+1280 t^{3} u^{3}+915 t^{2} u^{4}+303 t u^{5}+32 u^{6}\right)\right] \\
& -2 m_{h}^{6}\left[16 s^{7}+116 s^{6}(t+u)+s^{5}\left(297 t^{2}+602 t u+297 u^{2}\right)\right. \\
& +2 s^{4}(t+u)\left(197 t^{2}+418 t u+197 u^{2}\right) \\
& +s^{3}\left(335 t^{4}+1481 t^{3} u+2258 t^{2} u^{2}+1481 t u^{3}+335 u^{4}\right)
\end{aligned}
$$




$$
\begin{aligned}
& +2 s^{2}(t+u)\left(93 t^{4}+473 t^{3} u+723 t^{2} u^{2}+473 t u^{3}+93 u^{4}\right) \\
& +s\left(54 t^{6}+464 t^{5} u+1319 t^{4} u^{2}+1814 t^{3} u^{3}+1319 t^{2} u^{4}+464 t u^{5}+54 u^{6}\right) \\
& \left.+2(t+u)\left(3 t^{6}+40 t^{5} u+134 t^{4} u^{2}+186 t^{3} u^{3}+134 t^{2} u^{4}+40 t u^{5}+3 u^{6}\right)\right] \\
& +m_{h}^{4}\left[4 s^{8}+52 s^{7}(t+u)+s^{6}\left(189 t^{2}+394 t u+189 u^{2}\right)\right. \\
& +3 s^{5}(t+u)\left(101 t^{2}+230 t u+101 u^{2}\right) \\
& +2 s^{4}\left(135 t^{4}+626 t^{3} u+960 t^{2} u^{2}+626 t u^{3}+135 u^{4}\right) \\
& +s^{3}(t+u)\left(165 t^{4}+886 t^{3} u+1330 t^{2} u^{2}+886 t u^{3}+165 u^{4}\right) \\
& +s^{2}\left(72 t^{6}+623 t^{5} u+1733 t^{4} u^{2}+2368 t^{3} u^{3}+1733 t^{2} u^{4}+623 t u^{5}+72 u^{6}\right) \\
& +s(t+u)\left(16 t^{6}+185 t^{5} u+559 t^{4} u^{2}+768 t^{3} u^{3}+559 t^{2} u^{4}+185 t u^{5}+16 u^{6}\right) \\
& \left.+t^{8}+28 t^{7} u+160 t^{6} u^{2}+376 t^{5} u^{3}+490 t^{4} u^{4}+376 t^{3} u^{5}+160 t^{2} u^{6}+28 t u^{7}+u^{8}\right] \\
& -m_{h}^{2}\left[4 s^{8}(t+u)+s^{7}\left(27 t^{2}+58 t u+27 u^{2}\right)+2 s^{6}(t+u)\left(31 t^{2}+80 t u+31 u^{2}\right)\right. \\
& +s^{5}\left(67 t^{4}+354 t^{3} u+562 t^{2} u^{2}+354 t u^{3}+67 u^{4}\right) \\
& +4 s^{4}(t+u)\left(10 t^{4}+66 t^{3} u+101 t^{2} u^{2}+66 t u^{3}+10 u^{4}\right) \\
& +s^{3}(t+u)^{2}\left(17 t^{4}+153 t^{3} u+213 t^{2} u^{2}+153 t u^{3}+17 u^{4}\right) \\
& +2 s^{2}(t+u)\left(3 t^{6}+42 t^{5} u+122 t^{4} u^{2}+168 t^{3} u^{3}+122 t^{2} u^{4}+42 t u^{5}+3 u^{6}\right) \\
& +s(t+u)^{2}\left(t^{6}+21 t^{5} u+70 t^{4} u^{2}+94 t^{3} u^{3}+70 t^{2} u^{4}+21 t u^{5}+u^{6}\right) \\
& \left.+2 t u(t+u)\left(t^{2}+t u+u^{2}\right)\left(t^{4}+8 t^{3} u+12 t^{2} u^{2}+8 t u^{3}+u^{4}\right)\right] \\
& +(t+u)\left[s^{8}(t+u)+4 s^{7}\left(t^{2}+3 t u+u^{2}\right)+3 s^{6}(t+u)\left(2 t^{2}+9 t u+2 u^{2}\right)\right. \\
& +s^{5}\left(4 t^{4}+39 t^{3} u+66 t^{2} u^{2}+39 t u^{3}+4 u^{4}\right) \\
& +s^{4}(t+u)\left(t^{4}+24 t^{3} u+36 t^{2} u^{2}+24 t u^{3}+u^{4}\right) \\
& +2 s^{3} t u(t+u)^{2}\left(6 t^{2}+7 t u+6 u^{2}\right)+s^{2} t u(t+u)\left(5 t^{4}+15 t^{3} u+21 t^{2} u^{2}+15 t u^{3}+5 u^{4}\right) \\
& \left.\left.+s t u(t+u)^{2}\left(t^{4}+4 t^{3} u+5 t^{2} u^{2}+4 t u^{3}+u^{4}\right)+t^{2} u^{2}(t+u)\left(t^{2}+t u+u^{2}\right)^{2}\right]\right\}, \\
& \frac{d \sigma}{d t}\left(g g \rightarrow Q \bar{Q}\left[{ }^{3} P_{J}^{(8)}\right] h\right)=\frac{15}{8} \sum_{J^{\prime}=0}^{2}\left(2 J^{\prime}+1\right) \frac{d \sigma}{d t}\left(g g \rightarrow Q \bar{Q}\left[{ }^{3} P_{J^{\prime}}^{(1)}\right] h\right), \\
& \frac{d \sigma}{d t}\left(q \bar{q} \rightarrow Q \bar{Q}\left[{ }^{3} S_{1}^{(8)}\right] h\right)=\frac{4 \pi \alpha_{s}^{2}\left(y_{Q}^{h}\right)^{2}}{27 M s^{3}\left(2 m_{h}^{2}-t-u\right)^{2}} \\
& \times\left[2 m_{h}^{4}-2 m_{h}^{2}(3 s+t+u)+4 s^{2}+4 s(t+u)+t^{2}+u^{2}\right], \\
& \frac{d \sigma}{d t}\left(q \bar{q} \rightarrow Q \bar{Q}\left[{ }^{1}{ }^{(8)}\right] h\right)=\frac{16 \pi \alpha_{s}^{2}\left(y_{Q}^{h}\right)^{2}}{27 M^{3} s^{3}\left(2 m_{h}^{2}-t-u\right)^{2}} \\
& \times\left[2 m_{h}^{4}-2 m_{h}^{2}(s+t+u)+t^{2}+u^{2}\right], \\
& \frac{d \sigma}{d t}\left(\gamma g \rightarrow Q \bar{Q}\left[{ }^{1} S_{0}^{(8)}\right] h\right)=\frac{16 \alpha}{\alpha_{s}} \frac{d \sigma}{d t}\left(g g \rightarrow Q \bar{Q}\left[{ }^{1} S_{0}^{(1)}\right] h\right), \\
& \frac{d \sigma}{d t}\left(\gamma g \rightarrow Q \bar{Q}\left[{ }^{3} P_{J}^{(8)}\right] h\right)=\frac{16 \alpha}{\alpha_{s}} \sum_{J^{\prime}=0}^{2}\left(2 J^{\prime}+1\right) \frac{d \sigma}{d t}\left(g g \rightarrow Q \bar{Q}\left[{ }^{3} P_{J^{\prime}}^{(1)}\right] h\right),
\end{aligned}
$$




$$
\begin{aligned}
& \frac{d \sigma}{d t}(\gamma \gamma \rightarrow Q \bar{Q}[n] h)=\frac{512 \alpha^{2}}{9 \alpha_{s}^{2}} \frac{d \sigma}{d t}(g g \rightarrow Q \bar{Q}[n] h), \quad n={ }^{1} S_{0}^{(1)},{ }^{3} P_{J}^{(1)} \\
& \frac{d \sigma}{d t}\left(g g \rightarrow Q \bar{Q}\left[{ }^{1} S_{0}^{(1)}\right] A\right)=\frac{2 \pi \alpha_{s}^{2}\left(y_{Q}^{A}\right)^{2} M}{9 s^{2}\left(m_{A}^{2}-s-t\right)^{2}\left(m_{A}^{2}-s-u\right)^{2}\left(2 m_{A}^{2}-t-u\right)^{2}} \\
& \times\left\{m_{A}^{8}-2 m_{A}^{6}(s+t+u)+m_{A}^{4}\left[2 s^{2}+2 s(t+u)+t^{2}+4 t u+u^{2}\right]-2 m_{A}^{2} t u(s+t+u)\right. \\
& \left.+t^{2} u^{2}\right\} \\
& \frac{d \sigma}{d t}\left(g g \rightarrow Q \bar{Q}\left[{ }^{3} P_{J}^{(1)}\right] A\right)=\frac{-4 \pi \alpha_{s}^{2}\left(y_{Q}^{A}\right)^{2}}{135 M^{3} s^{2}\left(m_{A}^{2}-s-t\right)^{4}\left(m_{A}^{2}-s-u\right)^{4}\left(2 m_{A}^{2}-t-u\right)^{4}} \\
& \times G_{J}, \quad J=0,1,2, \\
& G_{0}=-10 M^{2}\left\{16 m_{A}^{16}(t-u)^{2}-16 m_{A}^{14}(t-u)^{2}[3 s+4(t+u)]\right. \\
& +4 m_{A}^{12}\left[s^{4}-4 s^{3}(t+u)+s^{2}\left(17 t^{2}-18 t u+17 u^{2}\right)+38 s(t+u)(t-u)^{2}\right. \\
& \left.+2(t-u)^{2}\left(13 t^{2}+30 t u+13 u^{2}\right)\right] \\
& -4 m_{A}^{10}\left[4 s^{5}-12 s^{4}(t+u)+2 s^{3}\left(3 t^{2}-2 t u+3 u^{2}\right)+s^{2}(t+u)\left(47 t^{2}-46 t u+47 u^{2}\right)\right. \\
& \left.+s(t-u)^{2}\left(47 t^{2}+110 t u+47 u^{2}\right)+2(t+u)(t-u)^{2}\left(11 t^{2}+34 t u+11 u^{2}\right)\right] \\
& +m_{A}^{8}\left[24 s^{6}-48 s^{5}(t+u)-72 s^{4}\left(t^{2}+t u+u^{2}\right)+20 s^{3}(t+u)\left(7 t^{2}+2 t u+7 u^{2}\right)\right. \\
& +3 s^{2}\left(71 t^{4}+88 t^{3} u+2 t^{2} u^{2}+88 t u^{3}+71 u^{4}\right)+6 s(t+u)(t-u)^{2}\left(19 t^{2}+62 t u+19 u^{2}\right) \\
& \left.+(t-u)^{2}\left(41 t^{4}+276 t^{3} u+486 t^{2} u^{2}+276 t u^{3}+41 u^{4}\right)\right] \\
& -2 m_{A}^{6}\left[8 s^{7}-8 s^{6}(t+u)-2 s^{5}\left(23 t^{2}+26 t u+23 u^{2}\right)+2 s^{4}(t+u)\left(5 t^{2}+12 t u+5 u^{2}\right)\right. \\
& +s^{3}\left(93 t^{4}+192 t^{3} u+230 t^{2} u^{2}+192 t u^{3}+93 u^{4}\right) \\
& +s^{2}(t+u)\left(65 t^{4}+96 t^{3} u-2 t^{2} u^{2}+96 t u^{3}+65 u^{4}\right) \\
& +s(t-u)^{2}\left(17 t^{4}+128 t^{3} u+230 t^{2} u^{2}+128 t u^{3}+17 u^{4}\right) \\
& \left.+(t+u)(t-u)^{2}\left(5 t^{4}+52 t^{3} u+110 t^{2} u^{2}+52 t u^{3}+5 u^{4}\right)\right] \\
& +m_{A}^{4}\left[4 s^{8}-12 s^{6}\left(3 t^{2}+4 t u+3 u^{2}\right)-4 s^{5}(t+u)\left(7 t^{2}+2 t u+7 u^{2}\right)\right. \\
& +2 s^{4}\left(37 t^{4}+96 t^{3} u+136 t^{2} u^{2}+96 t u^{3}+37 u^{4}\right) \\
& +2 s^{3}(t+u)\left(57 t^{4}+98 t^{3} u+130 t^{2} u^{2}+98 t u^{3}+57 u^{4}\right) \\
& +s^{2}\left(47 t^{6}+164 t^{5} u+197 t^{4} u^{2}+144 t^{3} u^{3}+197 t^{2} u^{4}+164 t u^{5}+47 u^{6}\right) \\
& +4 s(t+u)(t-u)^{2}\left(t^{4}+15 t^{3} u+34 t^{2} u^{2}+15 t u^{3}+u^{4}\right) \\
& \left.+\left(t^{2}-u^{2}\right)^{2}\left(t^{4}+22 t^{3} u+66 t^{2} u^{2}+22 t u^{3}+u^{4}\right)\right] \\
& +2 m_{A}^{2}(t+u)\left[2 s^{7}(t+u)+4 s^{6}\left(t^{2}+t u+u^{2}\right)-s^{5}(t+u)\left(5 t^{2}+8 t u+5 u^{2}\right)\right. \\
& -s^{4}\left(19 t^{4}+42 t^{3} u+58 t^{2} u^{2}+42 t u^{3}+19 u^{4}\right) \\
& -s^{3}(t+u)\left(17 t^{4}+23 t^{3} u+36 t^{2} u^{2}+23 t u^{3}+17 u^{4}\right) \\
& -s^{2}\left(5 t^{6}+15 t^{5} u+21 t^{4} u^{2}+14 t^{3} u^{3}+21 t^{2} u^{4}+15 t u^{5}+5 u^{6}\right) \\
& \left.-3 s t u(t+u)(t-u)^{2}\left(t^{2}+4 t u+u^{2}\right)-t u\left(t^{2}-u^{2}\right)^{2}\left(t^{2}+6 t u+u^{2}\right)\right] \\
& +(t+u)^{2}(s+t+u)^{2}\left[s^{4}(t+u)^{2}+2 s^{3}(t+u)\left(t^{2}+u^{2}\right)+s^{2}\left(t^{2}+u^{2}\right)^{2}\right. \\
& \left.\left.+t^{2} u^{2}(t-u)^{2}\right]\right\} \text {, }
\end{aligned}
$$




$$
\begin{aligned}
& G_{1}=5\left\{64 m_{A}^{22}-48 m_{A}^{20}[9 s+8(t+u)]\right. \\
& +4 m_{A}^{18}\left[325 s^{2}+584 s(t+u)+4\left(63 t^{2}+134 t u+63 u^{2}\right)\right] \\
& -4 m_{A}^{16}\left[566 s^{3}+1563 s^{2}(t+u)+s\left(1381 t^{2}+2890 t u+1381 u^{2}\right)\right. \\
& \left.+20(t+u)\left(19 t^{2}+46 t u+19 u^{2}\right)\right] \\
& +4 m_{A}^{14}\left[619 s^{4}+2376 s^{3}(t+u)+s^{2}\left(3267 t^{2}+6758 t u+3267 u^{2}\right)\right. \\
& +9 s(t+u)\left(209 t^{2}+478 t u+209 u^{2}\right) \\
& \left.+363 t^{4}+1796 t^{3} u+2882 t^{2} u^{2}+1796 t u^{3}+363 u^{4}\right] \\
& -2 m_{A}^{12}\left[864 s^{5}+4422 s^{4}(t+u)+s^{3}\left(8557 t^{2}+17582 t u+8557 u^{2}\right)\right. \\
& +4 s^{2}(t+u)\left(1951 t^{2}+4298 t u+1951 u^{2}\right) \\
& +s\left(3269 t^{4}+15024 t^{3} u+23558 t^{2} u^{2}+15024 t u^{3}+3269 u^{4}\right) \\
& \left.+8(t+u)\left(57 t^{4}+332 t^{3} u+566 t^{2} u^{2}+332 t u^{3}+57 u^{4}\right)\right] \\
& +m_{A}^{10}\left[748 s^{6}+5072 s^{5}(t+u)+2 s^{4}\left(6631 t^{2}+13590 t u+6631 u^{2}\right)\right. \\
& +2 s^{3}(t+u)\left(8637 t^{2}+18662 t u+8637 u^{2}\right) \\
& +s^{2}\left(11747 t^{4}+51776 t^{3} u+80050 t^{2} u^{2}+51776 t u^{3}+11747 u^{4}\right) \\
& +4 s(t+u)\left(946 t^{4}+4773 t^{3} u+7714 t^{2} u^{2}+4773 t u^{3}+946 u^{4}\right) \\
& \left.+(t+u)^{2}\left(377 t^{4}+2748 t^{3} u+5174 t^{2} u^{2}+2748 t u^{3}+377 u^{4}\right)\right] \\
& -m_{A}^{8}\left[184 s^{7}+1716 s^{6}(t+u)+16 s^{5}\left(378 t^{2}+775 t u+378 u^{2}\right)\right. \\
& +4 s^{4}(t+u)\left(2697 t^{2}+5798 t u+2697 u^{2}\right) \\
& +s^{3}\left(10665 t^{4}+46174 t^{3} u+70962 t^{2} u^{2}+46174 t u^{3}+10665 u^{4}\right) \\
& +s^{2}(t+u)\left(5765 t^{4}+27152 t^{3} u+42606 t^{2} u^{2}+27152 t u^{3}+5765 u^{4}\right) \\
& +s(t+u)^{2}\left(1471 t^{4}+8390 t^{3} u+14046 t^{2} u^{2}+8390 t u^{3}+1471 u^{4}\right) \\
& \left.+(t+u)^{3}\left(99 t^{4}+980 t^{3} u+2162 t^{2} u^{2}+980 t u^{3}+99 u^{4}\right)\right] \\
& +m_{A}^{6}\left[20 s^{8}+304 s^{7}(t+u)+80 s^{6}\left(19 t^{2}+39 t u+19 u^{2}\right)\right. \\
& +4 s^{5}(t+u)\left(927 t^{2}+2002 t u+927 u^{2}\right) \\
& +2 s^{4}\left(2549 t^{4}+11026 t^{3} u+16952 t^{2} u^{2}+11026 t u^{3}+2549 u^{4}\right) \\
& +8 s^{3}(t+u)\left(512 t^{4}+2357 t^{3} u+3666 t^{2} u^{2}+2357 t u^{3}+512 u^{4}\right) \\
& +s^{2}(t+u)^{2}\left(1829 t^{4}+9506 t^{3} u+15006 t^{2} u^{2}+9506 t u^{3}+1829 u^{4}\right) \\
& +2 s(t+u)\left(187 t^{6}+1640 t^{5} u+4899 t^{4} u^{2}+6900 t^{3} u^{3}+4899 t^{2} u^{4}+1640 t u^{5}+187 u^{6}\right) \\
& \left.+5(t+u)^{4}\left(3 t^{4}+46 t^{3} u+130 t^{2} u^{2}+46 t u^{3}+3 u^{4}\right)\right] \\
& -m_{A}^{4}\left[20 s^{8}(t+u)+2 s^{7}\left(89 t^{2}+182 t u+89 u^{2}\right)+4 s^{6}(t+u)\left(157 t^{2}+342 t u+157 u^{2}\right)\right. \\
& +4 s^{5}\left(301 t^{4}+1317 t^{3} u+2036 t^{2} u^{2}+1317 t u^{3}+301 u^{4}\right) \\
& +2 s^{4}(t+u)\left(689 t^{4}+3194 t^{3} u+5012 t^{2} u^{2}+3194 t u^{3}+689 u^{4}\right) \\
& +s^{3}\left(941 t^{6}+6676 t^{5} u+18033 t^{4} u^{2}+24588 t^{3} u^{3}+18033 t^{2} u^{4}+6676 t u^{5}+941 u^{6}\right) \\
& +s^{2}(t+u)\left(353 t^{6}+2874 t^{5} u+8015 t^{4} u^{2}+11020 t^{3} u^{3}+8015 t^{2} u^{4}+2874 t u^{5}+353 u^{6}\right) \\
& +s\left(57 t^{8}+738 t^{7} u+3246 t^{6} u^{2}+7294 t^{5} u^{3}+9442 t^{4} u^{4}\right. \\
& \left.+7294 t^{3} u^{5}+3246 t^{2} u^{6}+738 t u^{7}+57 u^{8}\right)
\end{aligned}
$$




$$
\begin{aligned}
& \left.+(t+u)^{5}\left(t^{4}+32 t^{3} u+134 t^{2} u^{2}+32 t u^{3}+u^{4}\right)\right] \\
& +m_{A}^{2}\left[6 s^{8}(t+u)^{2}+2 s^{7}(t+u)\left(19 t^{2}+42 t u+19 u^{2}\right)\right. \\
& +s^{6}\left(111 t^{4}+500 t^{3} u+782 t^{2} u^{2}+500 t u^{3}+111 u^{4}\right) \\
& +4 s^{5}(t+u)\left(47 t^{4}+225 t^{3} u+364 t^{2} u^{2}+225 t u^{3}+47 u^{4}\right) \\
& +2 s^{4}\left(96 t^{6}+691 t^{5} u+1909 t^{4} u^{2}+2624 t^{3} u^{3}+1909 t^{2} u^{4}+691 t u^{5}+96 u^{6}\right) \\
& +2 s^{3}(t+u)\left(57 t^{6}+464 t^{5} u+1294 t^{4} u^{2}+1778 t^{3} u^{3}+1294 t^{2} u^{4}+464 t u^{5}+57 u^{6}\right) \\
& +s^{2}\left(35 t^{8}+440 t^{7} u+1811 t^{6} u^{2}+3888 t^{5} u^{3}+4952 t^{4} u^{4}\right. \\
& \left.+3888 t^{3} u^{5}+1811 t^{2} u^{6}+440 t u^{7}+35 u^{8}\right) \\
& +4 s(t+u)\left(t^{8}+20 t^{7} u+93 t^{6} u^{2}+215 t^{5} u^{3}+278 t^{4} u^{4}\right. \\
& \left.\left.+215 t^{3} u^{5}+93 t^{2} u^{6}+20 t u^{7}+u^{8}\right)+t u(t+u)^{6}\left(2 t^{2}+17 t u+2 u^{2}\right)\right] \\
& -(t+u)\left[s^{7}(t+u)\left(t^{2}+4 t u+u^{2}\right)+s^{6}\left(5 t^{4}+28 t^{3} u+50 t^{2} u^{2}+28 t u^{3}+5 u^{4}\right)\right. \\
& +2 s^{5}(t+u)\left(5 t^{4}+28 t^{3} u+52 t^{2} u^{2}+28 t u^{3}+5 u^{4}\right) \\
& +2 s^{4}(t+u)^{2}\left(5 t^{4}+32 t^{3} u+56 t^{2} u^{2}+32 t u^{3}+5 u^{4}\right) \\
& +s^{3}(t+u)\left(5 t^{6}+56 t^{5} u+160 t^{4} u^{2}+224 t^{3} u^{3}+160 t^{2} u^{4}+56 t u^{5}+5 u^{6}\right) \\
& +s^{2}\left(t^{8}+24 t^{7} u+103 t^{6} u^{2}+224 t^{5} u^{3}+284 t^{4} u^{4}+224 t^{3} u^{5}+103 t^{2} u^{6}+24 t u^{7}+u^{8}\right) \\
& +s t u(t+u)\left(4 t^{6}+19 t^{5} u+46 t^{4} u^{2}+58 t^{3} u^{3}+46 t^{2} u^{4}+19 t u^{5}+4 u^{6}\right) \\
& \left.\left.+t^{2} u^{2}(t+u)^{6}\right]\right\} \\
& G_{2}=384 m_{A}^{22}-48 m_{A}^{20}[41 s+48(t+u)] \\
& +4 m_{A}^{18}\left[1071 s^{2}+2604 s(t+u)+4\left(379 t^{2}+802 t u+379 u^{2}\right)\right] \\
& -4 m_{A}^{16}\left[1350 s^{3}+4905 s^{2}(t+u)+s\left(6001 t^{2}+12634 t u+6001 u^{2}\right)\right. \\
& \left.+20(t+u)\left(115 t^{2}+274 t u+115 u^{2}\right)\right] \\
& +4 m_{A}^{14}\left[1201 s^{4}+5216 s^{3}(t+u)+s^{2}\left(9677 t^{2}+20274 t u+9677 u^{2}\right)\right. \\
& +6 s(t+u)\left(1319 t^{2}+3082 t u+1319 u^{2}\right) \\
& \left.+4\left(555 t^{4}+2696 t^{3} u+4298 t^{2} u^{2}+2696 t u^{3}+555 u^{4}\right)\right] \\
& -2 m_{A}^{12}\left[1768 s^{5}+7602 s^{4}(t+u)+s^{3}\left(17081 t^{2}+35646 t u+17081 u^{2}\right)\right. \\
& +8 s^{2}(t+u)\left(2706 t^{2}+6175 t u+2706 u^{2}\right) \\
& +3 s\left(4399 t^{4}+20772 t^{3} u+32874 t^{2} u^{2}+20772 t u^{3}+4399 u^{4}\right) \\
& \left.+16(t+u)\left(177 t^{4}+1000 t^{3} u+1678 t^{2} u^{2}+1000 t u^{3}+177 u^{4}\right)\right] \\
& +m_{A}^{10}\left[2116 s^{6}+8896 s^{5}(t+u)+2 s^{4}\left(9939 t^{2}+20686 t u+9939 u^{2}\right)\right. \\
& +2 s^{3}(t+u)\left(15713 t^{2}+34582 t u+15713 u^{2}\right) \\
& +s^{2}\left(30493 t^{4}+138040 t^{3} u+216542 t^{2} u^{2}+138040 t u^{3}+30493 u^{4}\right) \\
& +8 s(t+u)\left(1812 t^{4}+9605 t^{3} u+15890 t^{2} u^{2}+9605 t u^{3}+1812 u^{4}\right) \\
& \left.+2391 t^{6}+21478 t^{5} u+66169 t^{4} u^{2}+94100 t^{3} u^{3}+66169 t^{2} u^{4}+21478 t u^{5}+2391 u^{6}\right] \\
& -m_{A}^{8}\left[856 s^{7}+4028 s^{6}(t+u)+16 s^{5}\left(556 t^{2}+1153 t u+556 u^{2}\right)\right. \\
& +4 s^{4}(t+u)\left(3631 t^{2}+7486 t u+3631 u^{2}\right) \\
& +s^{3}\left(18407 t^{4}+76658 t^{3} u+118270 t^{2} u^{2}+76658 t u^{3}+18407 u^{4}\right)
\end{aligned}
$$




$$
\begin{aligned}
& +s^{2}(t+u)\left(14251 t^{4}+67500 t^{3} u+110090 t^{2} u^{2}+67500 t u^{3}+14251 u^{4}\right) \\
& +s\left(5289 t^{6}+43036 t^{5} u+127559 t^{4} u^{2}+179784 t^{3} u^{3}\right. \\
& \left.+127559 t^{2} u^{4}+43036 t u^{5}+5289 u^{6}\right) \\
& +(t+u)\left(645 t^{6}+7346 t^{5} u+25323 t^{4} u^{2}+37052 t^{3} u^{3}\right. \\
& \left.\left.+25323 t^{2} u^{4}+7346 t u^{5}+645 u^{6}\right)\right] \\
& +m_{A}^{6}\left[188 s^{8}+1104 s^{7}(t+u)+8 s^{6}\left(357 t^{2}+736 t u+357 u^{2}\right)\right. \\
& +8 s^{5}(t+u)\left(613 t^{2}+1150 t u+613 u^{2}\right) \\
& +s^{4}\left(7042 t^{4}+24892 t^{3} u+36808 t^{2} u^{2}+24892 t u^{3}+7042 u^{4}\right) \\
& +8 s^{3}(t+u)\left(932 t^{4}+3449 t^{3} u+5458 t^{2} u^{2}+3449 t u^{3}+932 u^{4}\right) \\
& +s^{2}\left(4531 t^{6}+30636 t^{5} u+84881 t^{4} u^{2}+117904 t^{3} u^{3}\right. \\
& \left.+84881 t^{2} u^{4}+30636 t u^{5}+4531 u^{6}\right) \\
& +6 s(t+u)\left(209 t^{6}+1962 t^{5} u+6259 t^{4} u^{2}+9092 t^{3} u^{3}+6259 t^{2} u^{4}+1962 t u^{5}+209 u^{6}\right) \\
& \left.+(t+u)^{2}\left(101 t^{6}+1664 t^{5} u+6795 t^{4} u^{2}+10240 t^{3} u^{3}+6795 t^{2} u^{4}+1664 t u^{5}+101 u^{6}\right)\right] \\
& -m_{A}^{4}\left[16 s^{9}+124 s^{8}(t+u)+2 s^{7}\left(229 t^{2}+470 t u+229 u^{2}\right)\right. \\
& +4 s^{6}(t+u)\left(261 t^{2}+446 t u+261 u^{2}\right) \\
& +4 s^{5}\left(464 t^{4}+1325 t^{3} u+1794 t^{2} u^{2}+1325 t u^{3}+464 u^{4}\right) \\
& +2 s^{4}(t+u)\left(1259 t^{4}+2936 t^{3} u+4152 t^{2} u^{2}+2936 t u^{3}+1259 u^{4}\right) \\
& +s^{3}\left(2151 t^{6}+10188 t^{5} u+23655 t^{4} u^{2}+31340 t^{3} u^{3}+23655 t^{2} u^{4}+10188 t u^{5}+2151 u^{6}\right) \\
& +s^{2}(t+u)\left(967 t^{6}+6318 t^{5} u+17545 t^{4} u^{2}+25076 t^{3} u^{3}\right. \\
& \left.+17545 t^{2} u^{4}+6318 t u^{5}+967 u^{6}\right) \\
& +s\left(179 t^{8}+2446 t^{7} u+11502 t^{6} u^{2}+27386 t^{5} u^{3}+36254 t^{4} u^{4}\right. \\
& \left.+27386 t^{3} u^{5}+11502 t^{2} u^{6}+2446 t u^{7}+179 u^{8}\right) \\
& \left.+(t+u)^{3}\left(7 t^{6}+226 t^{5} u+1225 t^{4} u^{2}+1884 t^{3} u^{3}+1225 t^{2} u^{4}+226 t u^{5}+7 u^{6}\right)\right] \\
& +m_{A}^{2}\left[14 s^{8}(t+u)^{2}+2 s^{7}(t+u)\left(39 t^{2}+58 t u+39 u^{2}\right)\right. \\
& +s^{6}\left(249 t^{4}+580 t^{3} u+674 t^{2} u^{2}+580 t u^{3}+249 u^{4}\right) \\
& +4 s^{5}(t+u)\left(127 t^{4}+174 t^{3} u+166 t^{2} u^{2}+174 t u^{3}+127 u^{4}\right) \\
& +2 s^{4}\left(306 t^{6}+935 t^{5} u+1375 t^{4} u^{2}+1480 t^{3} u^{3}+1375 t^{2} u^{4}+935 t u^{5}+306 u^{6}\right) \\
& +2 s^{3}(t+u)\left(201 t^{6}+738 t^{5} u+1426 t^{4} u^{2}+1854 t^{3} u^{3}+1426 t^{2} u^{4}+738 t u^{5}+201 u^{6}\right) \\
& +s^{2}\left(125 t^{8}+1044 t^{7} u+3733 t^{6} u^{2}+7996 t^{5} u^{3}+10328 t^{4} u^{4}\right. \\
& \left.+7996 t^{3} u^{5}+3733 t^{2} u^{6}+1044 t u^{7}+125 u^{8}\right) \\
& +4 s(t+u)\left(3 t^{8}+62 t^{7} u+308 t^{6} u^{2}+784 t^{5} u^{3}+1058 t^{4} u^{4}\right. \\
& \left.+784 t^{3} u^{5}+308 t^{2} u^{6}+62 t u^{7}+3 u^{8}\right) \\
& \left.+t u(t+u)^{4}\left(14 t^{4}+135 t^{3} u+206 t^{2} u^{2}+135 t u^{3}+14 u^{4}\right)\right] \\
& -(t+u)\left[s^{7}(t+u)\left(7 t^{2}-4 t u+7 u^{2}\right)+s^{6}\left(35 t^{4}+28 t^{3} u-2 t^{2} u^{2}+28 t u^{3}+35 u^{4}\right)\right. \\
& +2 s^{5}(t+u)\left(35 t^{4}+12 t^{3} u-8 t^{2} u^{2}+12 t u^{3}+35 u^{4}\right) \\
& +2 s^{4}(t+u)^{2}\left(35 t^{4}+8 t^{3} u+6 t^{2} u^{2}+8 t u^{3}+35 u^{4}\right)
\end{aligned}
$$




$$
\begin{aligned}
& +s^{3}(t+u)\left(35 t^{6}+104 t^{5} u+132 t^{4} u^{2}+176 t^{3} u^{3}+132 t^{2} u^{4}+104 t u^{5}+35 u^{6}\right) \\
& +s^{2}\left(7 t^{8}+64 t^{7} u+197 t^{6} u^{2}+420 t^{5} u^{3}+548 t^{4} u^{4}+420 t^{3} u^{5}+197 t^{2} u^{6}+64 t u^{7}+7 u^{8}\right) \\
& +3 s t u(t+u)\left(4 t^{6}+19 t^{5} u+54 t^{4} u^{2}+74 t^{3} u^{3}+54 t^{2} u^{4}+19 t u^{5}+4 u^{6}\right) \\
& \left.+t^{2} u^{2}(t+u)^{4}\left(7 t^{2}+10 t u+7 u^{2}\right)\right] \text {, } \\
& \frac{d \sigma}{d t}\left(g g \rightarrow Q \bar{Q}\left[{ }^{1} S_{0}^{(8)}\right] A\right)=\frac{15}{8} \frac{d \sigma}{d t}\left(g g \rightarrow Q \bar{Q}\left[{ }^{1} S_{0}^{(1)}\right] A\right) \\
& \frac{d \sigma}{d t}\left(g g \rightarrow Q \bar{Q}\left[{ }^{3} S_{1}^{(8)}\right] A\right)=\frac{\pi \alpha_{s}^{2}\left(y_{Q}^{A}\right)^{2}}{4 M s^{3}\left(m_{A}^{2}-s-t\right)^{2}\left(m_{A}^{2}-s-u\right)^{2}\left(2 m_{A}^{2}-t-u\right)^{2}} \\
& \times\left\{9 m_{A}^{12}-m_{A}^{10}[20 s+27(t+u)]+m_{A}^{8}\left[14 s^{2}+46 s(t+u)+3\left(11 t^{2}+23 t u+11 u^{2}\right)\right]\right. \\
& -m_{A}^{6}\left[3 s^{3}+23 s^{2}(t+u)+42 s(t+u)^{2}+3(t+u)\left(7 t^{2}+16 t u+7 u^{2}\right)\right] \\
& +m_{A}^{4}\left[3 s^{3}(t+u)+2 s^{2}\left(7 t^{2}+13 t u+7 u^{2}\right)+2 s(t+u)\left(9 t^{2}+20 t u+9 u^{2}\right)\right. \\
& \left.+7 t^{4}+35 t^{3} u+51 t^{2} u^{2}+35 t u^{3}+7 u^{4}\right] \\
& -m_{A}^{2}\left[s^{3}\left(t^{2}+t u+u^{2}\right)+s^{2}(t+u)\left(3 t^{2}+7 t u+3 u^{2}\right)\right. \\
& \left.+s\left(3 t^{4}+18 t^{3} u+26 t^{2} u^{2}+18 t u^{3}+3 u^{4}\right)+(t+u)\left(t^{2}+t u+u^{2}\right)\left(t^{2}+7 t u+u^{2}\right)\right] \\
& \left.+t u\left[s(t+u)+t^{2}+t u+u^{2}\right]^{2}\right\}, \\
& \frac{d \sigma}{d t}\left(g g \rightarrow Q \bar{Q}\left[{ }^{1} P_{1}^{(8)}\right] A\right)=\frac{\pi \alpha_{s}^{2}\left(y_{Q}^{A}\right)^{2}}{M^{3} s^{3}\left(m_{A}^{2}-s-t\right)^{3}\left(m_{A}^{2}-s-u\right)^{3}\left(2 m_{A}^{2}-t-u\right)^{4}} \\
& \times\left\{3 m_{A}^{16}\left[4 s^{2}+4 s(t+u)+3(t+u)^{2}\right]\right. \\
& -m_{A}^{14}\left[28 s^{3}+76 s^{2}(t+u)+s\left(73 t^{2}+142 t u+73 u^{2}\right)+36(t+u)^{3}\right] \\
& +m_{A}^{12}\left[28 s^{4}+108 s^{3}(t+u)+2 s^{2}\left(97 t^{2}+204 t u+97 u^{2}\right)\right. \\
& \left.+s(t+u)\left(161 t^{2}+330 t u+161 u^{2}\right)+12(t+u)^{2}\left(5 t^{2}+11 t u+5 u^{2}\right)\right] \\
& -m_{A}^{10}\left[20 s^{5}+76 s^{4}(t+u)+4 s^{3}\left(43 t^{2}+97 t u+43 u^{2}\right)\right. \\
& +2 s^{2}(t+u)\left(127 t^{2}+302 t u+127 u^{2}\right) \\
& \left.+s\left(181 t^{4}+783 t^{3} u+1192 t^{2} u^{2}+783 t u^{3}+181 u^{4}\right)+18(t+u)^{3}\left(3 t^{2}+8 t u+3 u^{2}\right)\right] \\
& +m_{A}^{8}\left[8 s^{6}+40 s^{5}(t+u)+s^{4}\left(79 t^{2}+190 t u+79 u^{2}\right)\right. \\
& +2 s^{3}(t+u)\left(73 t^{2}+206 t u+73 u^{2}\right)+4 s^{2}\left(48 t^{4}+229 t^{3} u+363 t^{2} u^{2}+229 t u^{3}+48 u^{4}\right) \\
& +s(t+u)\left(117 t^{4}+560 t^{3} u+866 t^{2} u^{2}+560 t u^{3}+117 u^{4}\right) \\
& \left.+2(t+u)^{2}\left(14 t^{4}+79 t^{3} u+129 t^{2} u^{2}+79 t u^{3}+14 u^{4}\right)\right] \\
& -m_{A}^{6}\left[8 s^{6}(t+u)+s^{5}\left(23 t^{2}+58 t u+23 u^{2}\right)+2 s^{4}(t+u)\left(19 t^{2}+66 t u+19 u^{2}\right)\right. \\
& +2 s^{3}\left(38 t^{4}+201 t^{3} u+340 t^{2} u^{2}+201 t u^{3}+38 u^{4}\right) \\
& +2 s^{2}(t+u)\left(45 t^{4}+226 t^{3} u+384 t^{2} u^{2}+226 t u^{3}+45 u^{4}\right) \\
& +s\left(45 t^{6}+337 t^{5} u+934 t^{4} u^{2}+1272 t^{3} u^{3}+934 t^{2} u^{4}+337 t u^{5}+45 u^{6}\right) \\
& \left.+4(t+u)^{3}\left(2 t^{4}+16 t^{3} u+27 t^{2} u^{2}+16 t u^{3}+2 u^{4}\right)\right] \\
& +m_{A}^{4}\left[2 s^{6}(t+u)^{2}+s^{5}(t+u)\left(5 t^{2}+14 t u+5 u^{2}\right)\right. \\
& +2 s^{4}\left(7 t^{4}+31 t^{3} u+58 t^{2} u^{2}+31 t u^{3}+7 u^{4}\right)
\end{aligned}
$$




$$
\begin{aligned}
& +s^{3}(t+u)\left(29 t^{4}+122 t^{3} u+262 t^{2} u^{2}+122 t u^{3}+29 u^{4}\right) \\
& +s^{2}\left(27 t^{6}+180 t^{5} u+539 t^{4} u^{2}+768 t^{3} u^{3}+539 t^{2} u^{4}+180 t u^{5}+27 u^{6}\right) \\
& +s(t+u)\left(10 t^{6}+86 t^{5} u+255 t^{4} u^{2}+342 t^{3} u^{3}+255 t^{2} u^{4}+86 t u^{5}+10 u^{6}\right) \\
& \left.+(t+u)^{2}\left(t^{6}+18 t^{5} u+63 t^{4} u^{2}+88 t^{3} u^{3}+63 t^{2} u^{4}+18 t u^{5}+u^{6}\right)\right] \\
& -m_{A}^{2}\left[s^{5}\left(t^{4}+t^{3} u+t u^{3}+u^{4}\right)+4 s^{4}(t+u)\left(t^{4}+2 t^{3} u+5 t^{2} u^{2}+2 t u^{3}+u^{4}\right)\right. \\
& +2 s^{3}\left(3 t^{6}+16 t^{5} u+48 t^{4} u^{2}+76 t^{3} u^{3}+48 t^{2} u^{4}+16 t u^{5}+3 u^{6}\right) \\
& +2 s^{2}(t+u)\left(2 t^{6}+15 t^{5} u+43 t^{4} u^{2}+72 t^{3} u^{3}+43 t^{2} u^{4}+15 t u^{5}+2 u^{6}\right) \\
& +s\left(t^{8}+15 t^{7} u+64 t^{6} u^{2}+142 t^{5} u^{3}+180 t^{4} u^{4}+142 t^{3} u^{5}+64 t^{2} u^{6}+15 t u^{7}+u^{8}\right) \\
& \left.+2 t u(t+u)^{3}\left(t^{4}+5 t^{3} u+6 t^{2} u^{2}+5 t u^{3}+u^{4}\right)\right] \\
& +t u(t+u)\left[s^{4}(t+u)\left(t^{2}+t u+u^{2}\right)+s^{3}\left(3 t^{4}+6 t^{3} u+14 t^{2} u^{2}+6 t u^{3}+3 u^{4}\right)\right. \\
& +s^{2}(t+u)\left(3 t^{4}+4 t^{3} u+14 t^{2} u^{2}+4 t u^{3}+3 u^{4}\right) \\
& \left.\left.+s(t+u)^{2}\left(t^{4}+2 t^{3} u+4 t^{2} u^{2}+2 t u^{3}+u^{4}\right)+t u(t+u)\left(t^{2}+t u+u^{2}\right)^{2}\right]\right\}, \\
& \frac{d \sigma}{d t}\left(g g \rightarrow Q \bar{Q}\left[{ }^{3} P_{J}^{(8)}\right] A\right)=\frac{15}{8} \sum_{J^{\prime}=0}^{2}\left(2 J^{\prime}+1\right) \frac{d \sigma}{d t}\left(g g \rightarrow Q \bar{Q}\left[{ }^{3} P_{J^{\prime}}^{(1)}\right] A\right), \\
& \frac{d \sigma}{d t}\left(q \bar{q} \rightarrow Q \bar{Q}\left[{ }^{3} S_{1}^{(8)}\right] A\right)=\frac{4 \pi \alpha_{s}^{2}\left(y_{Q}^{A}\right)^{2}}{27 M s^{3}\left(2 m_{A}^{2}-t-u\right)^{2}} \\
& \times\left[2 m_{A}^{4}-2 m_{A}^{2}(s+t+u)+t^{2}+u^{2}\right], \\
& \frac{d \sigma}{d t}\left(q \bar{q} \rightarrow Q \bar{Q}\left[{ }^{1} P_{1}^{(8)}\right] A\right)=\frac{-16 \pi \alpha_{s}^{2}\left(y_{Q}^{A}\right)^{2}}{27 M^{3} s^{3}\left(2 m_{A}^{2}-t-u\right)^{4}} \\
& \times\left\{8 m_{A}^{6} s-2 m_{A}^{4}(2 s+t+u)^{2}-2 m_{A}^{2}\left[s(t-u)^{2}-(t+u)^{3}\right]-(t+u)^{2}\left(t^{2}+u^{2}\right)\right\}, \\
& \frac{d \sigma}{d t}\left(\gamma g \rightarrow Q \bar{Q}\left[{ }^{1} S_{0}^{(8)}\right] A\right)=\frac{16 \alpha}{\alpha_{s}} \frac{d \sigma}{d t}\left(g g \rightarrow Q \bar{Q}\left[{ }^{1} S_{0}^{(1)}\right] A\right) \text {, } \\
& \frac{d \sigma}{d t}\left(\gamma g \rightarrow Q \bar{Q}\left[{ }^{3} P_{J}^{(8)}\right] A\right)=\frac{16 \alpha}{\alpha_{s}} \sum_{J^{\prime}=0}^{2}\left(2 J^{\prime}+1\right) \frac{d \sigma}{d t}\left(g g \rightarrow Q \bar{Q}\left[{ }^{3} P_{J^{\prime}}^{(1)}\right] A\right), \\
& \frac{d \sigma}{d t}(\gamma \gamma \rightarrow Q \bar{Q}[n] A)=\frac{512 \alpha^{2}}{9 \alpha_{s}^{2}} \frac{d \sigma}{d t}(g g \rightarrow Q \bar{Q}[n] A), \quad n={ }^{1} S_{0}^{(1)},{ }^{3} P_{J}^{(1)}
\end{aligned}
$$




\section{References}

[1] W.E. Caswell and G.P. Lepage, Phys. Lett. 167B, 437 (1986); G.T. Bodwin, E. Braaten, and G.P. Lepage, Phys. Rev. D 51, 1125 (1995); 55, 5853(E) (1997).

[2] E.L. Berger and D. Jones, Phys. Rev. D 23, 1521 (1981); R. Baier and R. Rückl, Phys. Lett. 102B, 364 (1981); Z. Phys. C 19, 251 (1983); B. Humpert, Phys. Lett. B 184, 105 (1987); R. Gastmans, W. Troost, and T.T. Wu, ibid. 184, 257 (1987); Nucl. Phys. B291, 731 (1987).

[3] E. Braaten and S. Fleming, Phys. Rev. Lett. 74, 3327 (1995); E. Braaten and T.C. Yuan, Phys. Rev. D 52, 6627 (1995).

[4] P. Cho and A.K. Leibovich, Phys. Rev. D 53, 150 (1996).

[5] P. Cho and A.K. Leibovich, Phys. Rev. D 53, 6203 (1996).

[6] CDF Collaboration, F. Abe et al., Phys. Rev. Lett. 69, 3704 (1992); 71, 2537 (1993); 79, 572 (1997); 79, 578 (1997); D0 Collaboration, S. Abachi et al., Phys. Lett. B 370, 239 (1996); D0 Collaboration, B. Abbott et al., Phys. Rev. Lett. 82, 35 (1999).

[7] R. Barbieri, R. Gatto, and E. Remiddi, Phys. Lett. 61B, 465 (1976).

[8] K. Sridhar, A.D. Martin, and W.J. Stirling, Phys. Lett. B 438, 211 (1998); Ph. Hägler, R. Kirschner, A. Schäfer, L. Szymanowski, and O.V. Teryaev, Phys. Rev. Lett. 86, 1446 (2001); Phys. Rev. D 63, 077501 (2001); F. Yuan and K.-T. Chao, ibid. 63, 034006 (2001); Phys. Rev. Lett. 87, 022002 (2001); V.A. Saleev, Phys. Rev. D 65, 054041 (2002); S.P. Baranov, ibid. 66, 114003 (2002); V.A. Saleev and D.V. Vasin, Phys. Lett. B 548, 161 (2002); A.V. Lipatov and N.P. Zotov, Eur. Phys. J. C 27, 87 (2003).

[9] P. Hoyer and S. Peigné, Phys. Rev. D 59, 034011 (1999); N. Marchal, S. Peigné, and P. Hoyer, ibid. 62, 114001 (2000).

[10] H. Fritzsch, Phys. Lett. 67B, 217 (1977); F. Halzen, ibid. 69B, 105 (1977); M. Glück, J.F. Owens, and E. Reya, Phys. Rev. D 17, 2324 (1978).

[11] G.A. Schuler and R. Vogt, Phys. Lett. B 387, 181 (1996); J.F. Amundson, O.J.P. Eboli, E.M. Gregores, and F. Halzen, ibid. B 390, 323 (1997).

[12] E. Braaten, S. Fleming, and T.C. Yuan, Annu. Rev. Nucl. Part. Sci. 46, 197 (1996); B.A. Kniehl and G. Kramer, Phys. Lett. B 413, 416 (1997).

[13] M. Krämer, Prog. Part. Nucl. Phys. 47, 141 (2001).

[14] M. Beneke and M. Krämer, Phys. Rev. D 55, 5269 (1997); A.K. Leibovich, ibid. 56, 4412 (1997); M. Beneke, M. Krämer, and M. Vänttinen, ibid. 57, 4258 (1998); S. Fleming, I.Z. Rothstein, and A.K. Leibovich, ibid. 64, 036002 (2001). 
[15] E. Braaten, B.A. Kniehl, and J. Lee, Phys. Rev. D 62, 094005 (2000).

[16] B.A. Kniehl and J. Lee, Phys. Rev. D 62, 114027 (2000); B.A. Kniehl, G. Kramer, and C.P. Palisoc, ibid. 68, 114002 (2003).

[17] H1 Collaboration, C. Adloff et al., Eur. Phys. J. C 10, 373 (1999); 25, 41 (2002).

[18] DELPHI Collaboration, J. Abdallah et al., Phys. Lett. B 565, 76 (2003).

[19] B.A. Kniehl and L. Zwirner, Nucl. Phys. B621, 337 (2002).

[20] M. Klasen, B.A. Kniehl, L.N. Mihaila, and M. Steinhauser, Phys. Rev. Lett. 89, 032001 (2002).

[21] B.A. Kniehl, C.P. Palisoc, and L. Zwirner, Phys. Rev. D 66, 114002 (2002).

[22] V. Barger, S. Fleming, and R.J.N. Phillips, Phys. Lett. B 371, 111 (1996).

[23] E. Braaten, J. Lee, and S. Fleming, Phys. Rev. D 60, 091501(R) (1999).

[24] CDF Collaboration, D. Acosta et al., Phys. Rev. Lett. 90, 221803 (2003).

[25] E.J. Eichten and C. Quigg, Phys. Rev. D 52, 1726 (1995).

[26] M. Spira, Fortschr. Phys. 46, 203 (1998).

[27] A. Petrelli, M. Cacciari, M. Greco, F. Maltoni, and M.L. Mangano, Nucl. Phys. B514, 245 (1998); F. Maltoni, M.L. Mangano, and A. Petrelli, ibid. B519, 361 (1998).

[28] Particle Data Group, K. Hagiwara et al., Phys. Rev. D 66, 010001 (2002).

[29] CTEQ Collaboration, H.L. Lai et al., Eur. Phys. J. C 12, 375 (2000).

[30] W. Buchmüller and S.-H.H. Tye, Phys. Rev. D 24, 132 (1981).

[31] CDF Collaboration, D. Acosta et al., Phys. Rev. Lett. 88, 161802 (2002).

[32] B.A. Kniehl and L. Zwirner, Nucl. Phys. B637, 311 (2002).

[33] A. Martin, Phys. Lett. 93B, 338 (1980).

[34] C. Quigg and J.L. Rosner, Phys. Lett. 71B, 153 (1977).

[35] E. Eichten, K. Gottfried, T. Kinoshita, K.D. Lane, and T.-M. Yan, Phys. Rev. D 17, 3090 (1978); 21, 313(E) (1980); 21, 203 (1980).

[36] CDF Collaboration, F. Abe et al., Phys. Rev. Lett. 75, 4358 (1995).

[37] M. Witherell, talk presented on 14 January 2004 at the Collider Physics Conference, KITP, Santa Barbara, California. 


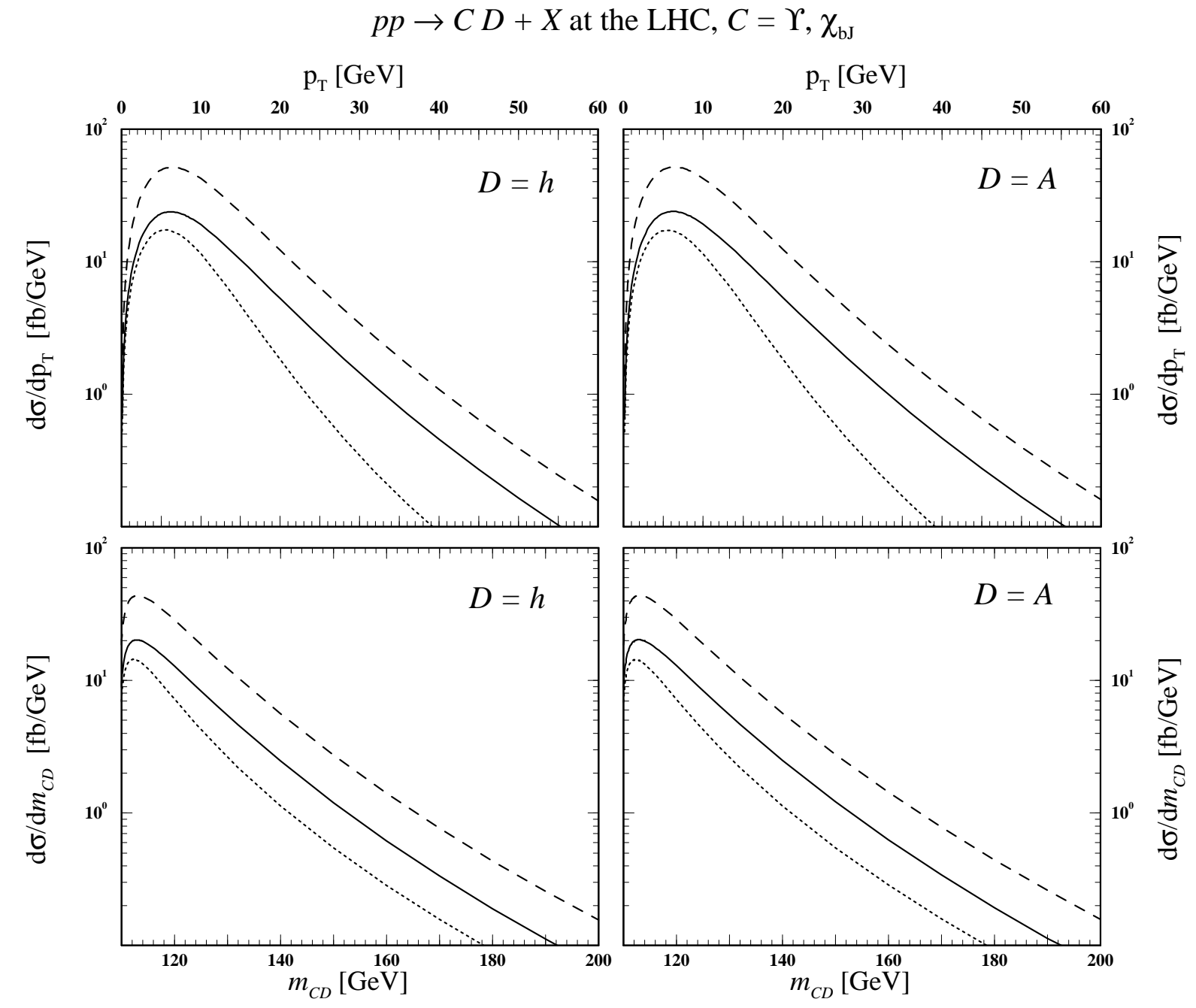

(a)

Figure 1: (a) $p_{T}$ distributions $d \sigma / d p_{T}$ (upper panel) and $m_{C D}$ distributions $d \sigma / d m_{C D}$ (lower panel) in $\mathrm{fb} / \mathrm{GeV}$, (b) $y_{C}$ distributions $d \sigma / d y_{C}$ (upper panel) and $y_{D}$ distributions $d \sigma / d y_{D}$ (lower panel) in $\mathrm{fb}$, and (c) total cross sections $\sigma$ in $\mathrm{fb}$ as functions of $\tan \beta$ (upper panel) and $m_{D}$ (lower panel) of $p p \rightarrow C D+X$, where $C=\Upsilon(1 S), \chi_{b J}(1 P)$ and $D=h$ (left columns) or $D=A$ (right columns), at the LHC. The default values of the MSSM input parameters are $\tan \beta=50$ and $m_{D}=100 \mathrm{GeV}$. It is summed over $C=\chi_{b 0}(1 P), \chi_{b 1}(1 P), \chi_{b 2}(1 P)$. In each frame, the NRQCD (solid line) prediction for $C=\Upsilon(1 S)$ and the NRQCD (dashed line) and CSM (dotted line) ones for $C=\chi_{b J}(1 P)$ are shown separately. 


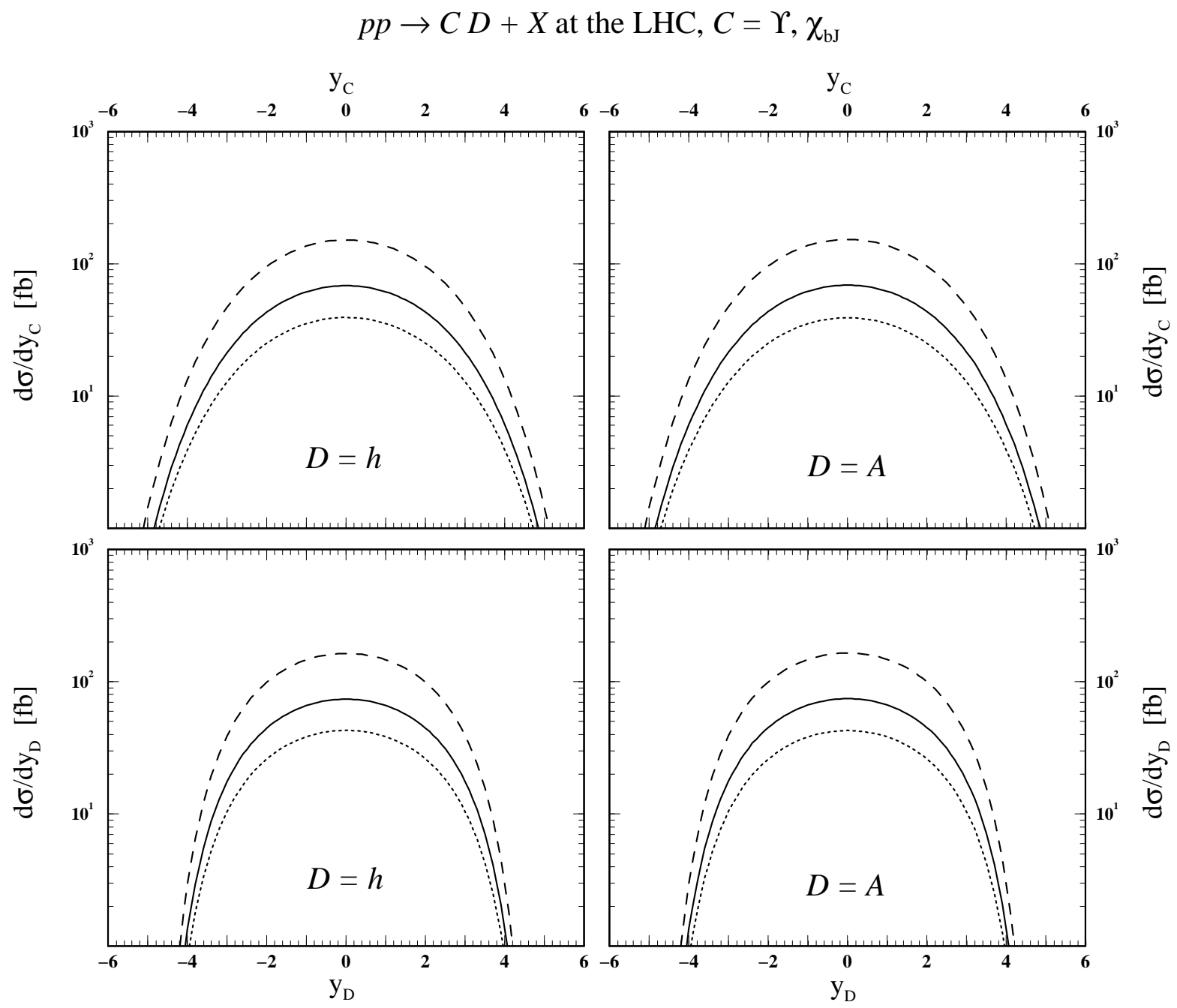

(b)

Fig. 1 (continued). 


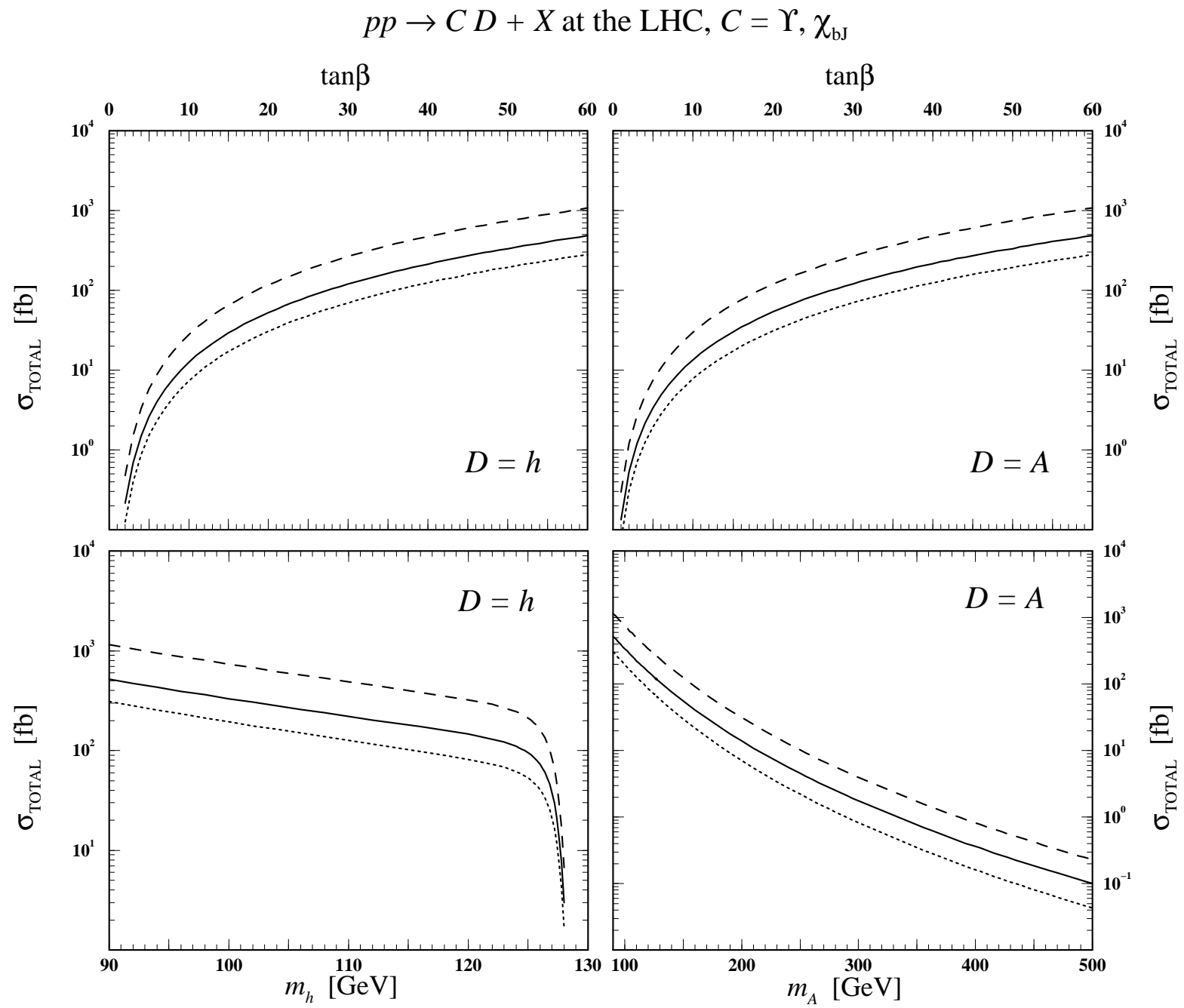

(c)

Fig. 1 (continued). 


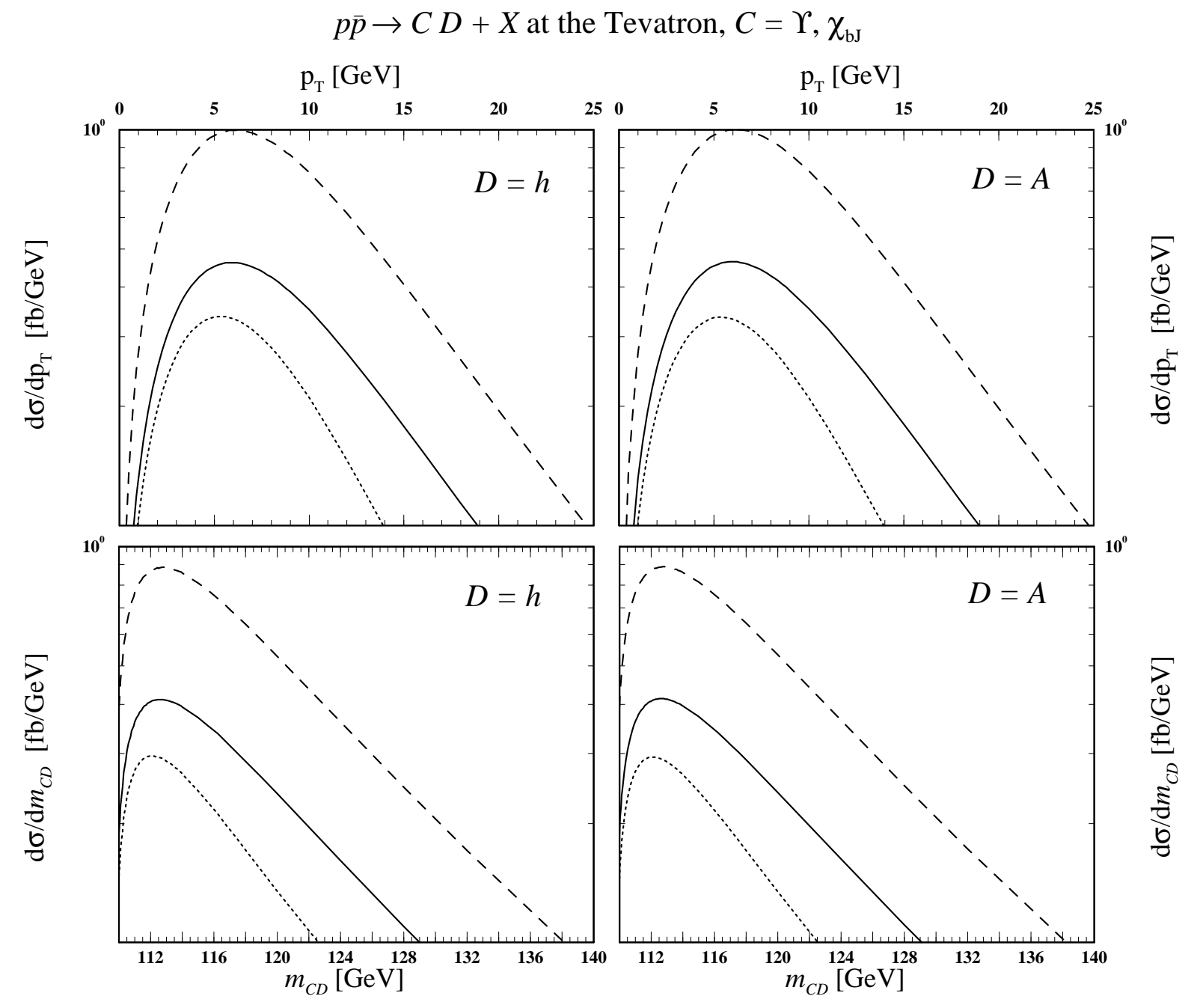

(a)

Figure 2: Same as in Figs. 1(a)-(c), but for $p \bar{p} \rightarrow C D+X$ in Run II at the Tevatron. 


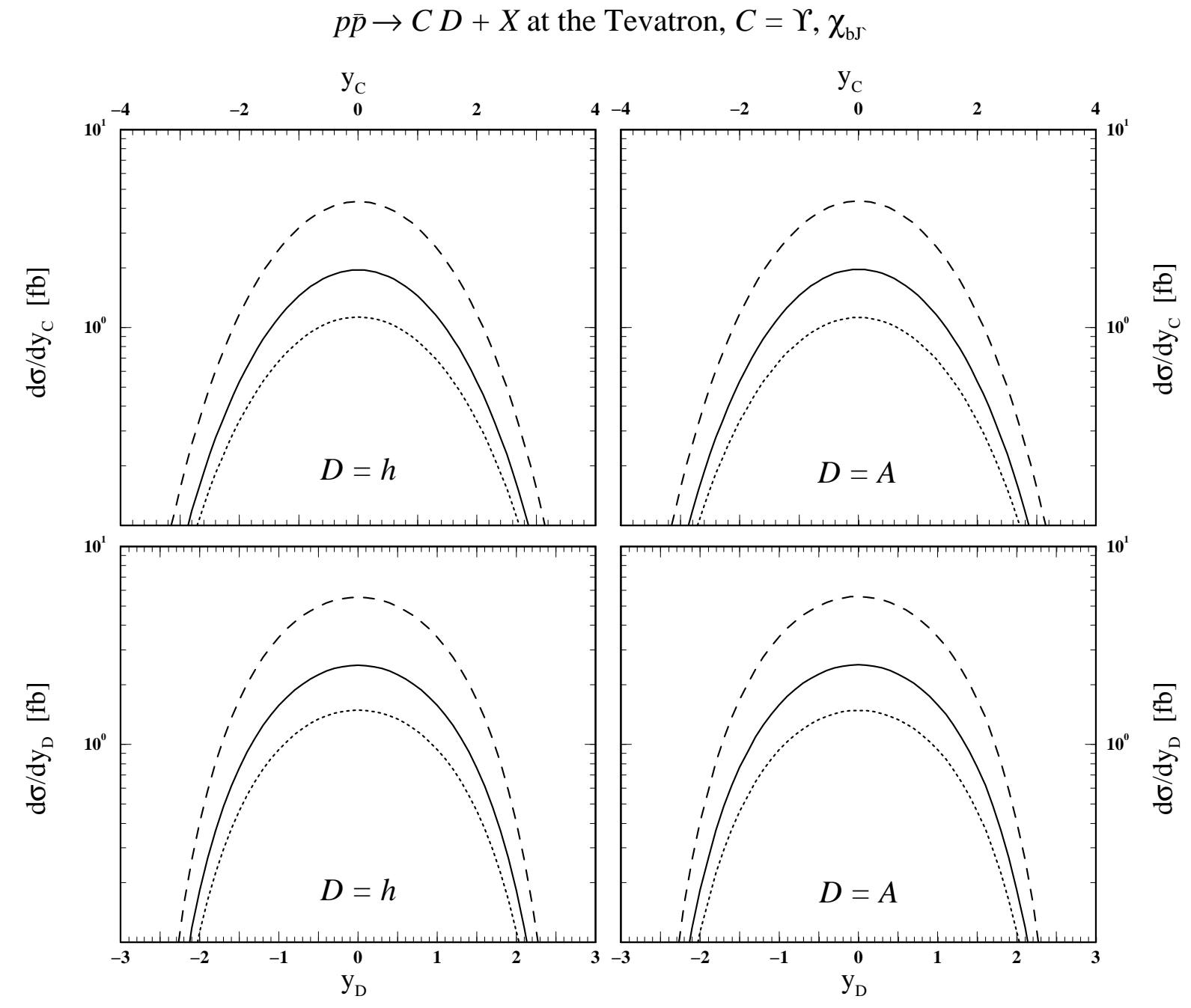

(b)

Fig. 2 (continued). 


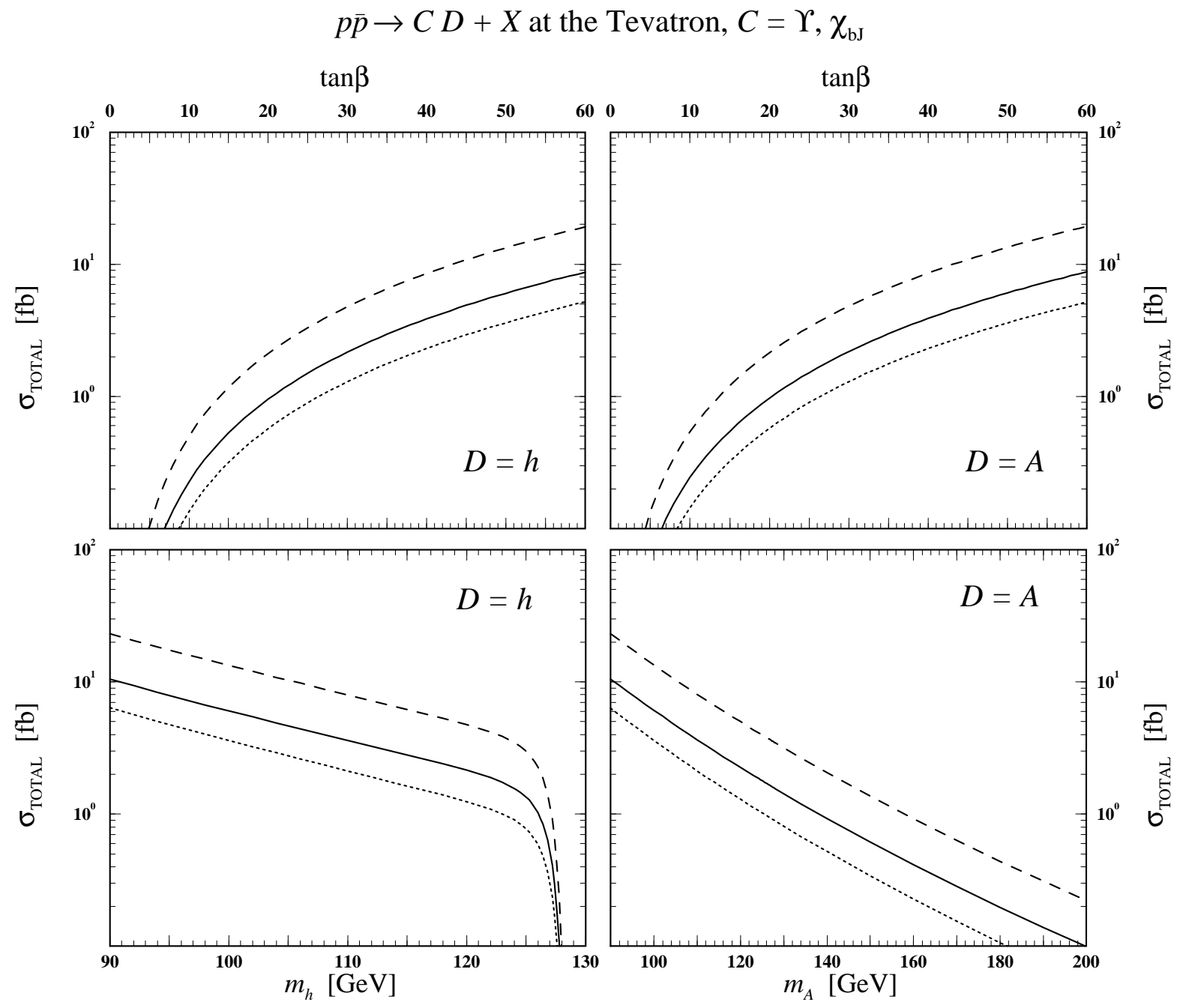

(c)

Fig. 2 (continued). 


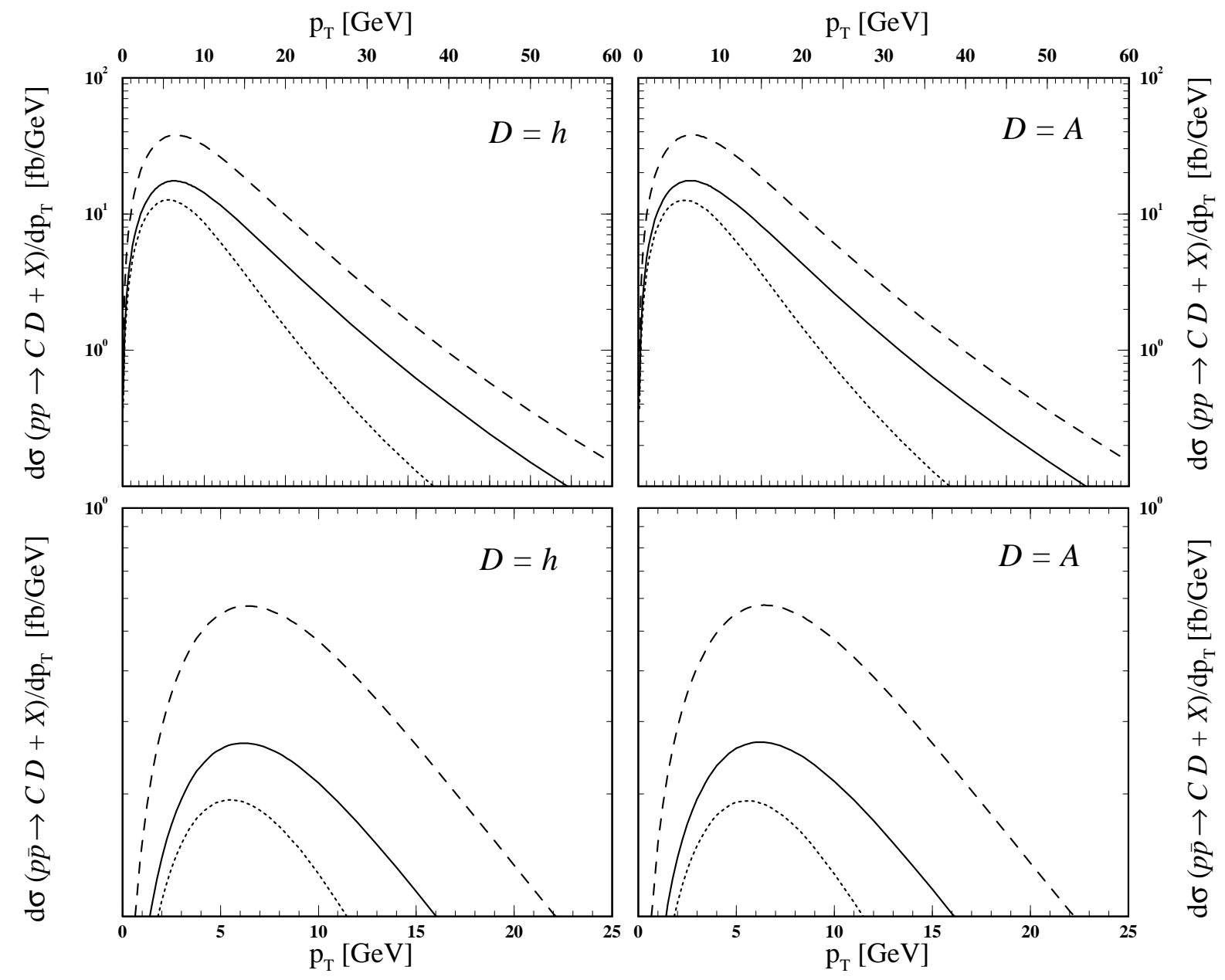

Figure 3: Same as in the upper panels of Figs. 1(a) and Figs. 2(a), but for $\mu_{r}=\mu_{f}=m_{D}$. 